\title{
Structures of an intact yeast V-ATPase alone and in complex with bacterial
}

\section{effector VopQ}




\begin{abstract}
Vacuolar-type ATPase (V-ATPase) is a rotary protein pump involved in proton translocation across various cellular membranes using the energy of ATP hydrolysis. Despite previous studies on bacterial and eukaryotic V-ATPases, information on the intact structure of a eukaryotic VATPase is missing. Here we report cryo-EM structures of the intact yeast V-ATPase and this complex bound to a bacterial effector. We reveal the interaction of the elusive regulatory subunit $\mathrm{H}$ with its neighboring subunits. Insight for the catalysis mechanism is gained by determining conformations of the catalytic subunits either empty or bound with nucleotides.
\end{abstract}

\title{
Introduction
}

V-ATPase is a giant protein complex with the primary function of pumping protons into organelles to create a local acidic environment and membrane potential for biological processes such as protein degradation in lysosome ${ }^{1}$. As an important protein machine present in various membranes, V-ATPase is also involved in other cellular functions exemplified by mTOR signaling, Notch signaling, neurotransmitter release, and bone remodeling ${ }^{2-5}$. Consequently, dysfunction of V-ATPase is associated with related diseases.

V-ATPase is composed of 16 known unique subunits in yeast and mammals with 31 and 32 polypeptides, resulting in a molecular mass of around 1 megadalton ${ }^{6-8}$. The proton translocation is built on the coupling between ATP hydrolysis within the cytosolic $\mathrm{V}_{1}$ subcomplex and proton transport within the membrane-inserted $\mathrm{V}_{\mathrm{o}}$ subcomplex ${ }^{9,10}$. The $\mathrm{V}_{1}$ subcomplex contains subunits of $\mathrm{A}_{3}, \mathrm{~B}_{3}, \mathrm{C}, \mathrm{D}, \mathrm{E}_{3}, \mathrm{~F}, \mathrm{G}_{3}$, and $\mathrm{H}$ and the $\mathrm{V}_{\mathrm{o}}$ subcomplex includes 
subunits of a, $\mathrm{c}_{8}, \mathrm{c}^{\prime}, \mathrm{c}^{\prime \prime}, \mathrm{d}, \mathrm{e}, \mathrm{f}$, and Voa1 in yeast or a, $\mathrm{c}_{9}, \mathrm{c}^{\prime \prime}, \mathrm{d}, \mathrm{e}, \mathrm{f}, \mathrm{Ac} 45$, and PRR in mammals ${ }^{6-8}$. As a member of the rotary ATPases, V-ATPase shares similarities in the architecture and working mechanism with F-ATPase (F-type) and A-ATPase (archaeal type) ${ }^{11-13}$. However, V-ATPase still differs in many ways from the well-studied F-ATPase or A-ATPase that usually catalyze ATP synthesis driven by protonmotive force. Major differences in VATPase include three copies of peripheral stalk subunits $\left(\mathrm{E}_{3} \mathrm{G}_{3}\right)$ instead of one (or two in AATPase) and additional subunits of $\mathrm{C}$ and $\mathrm{H}$, larger c-ring subunit scaffold (two helix hairpins containing four helices), regulated disassembly in response to environmental factors, and the catalysis of ATP hydrolysis instead of ATP synthesis ${ }^{10,11}$.

Recent progress on bacterial V/A-ATPases and eukaryotic V-ATPases has begun to shed light on the structures and mechanisms of V-ATPase - $^{7,8,14-17}$. Although structures of a rat VATPase were reported recently ${ }^{8}$, the rat V-ATPase is not an intact holoenzyme as it lacks the regulatory subunit $\mathrm{H}$. Herein, we describe cryo-EM structures of the first intact eukaryotic holoenzyme V-ATPase complex $\left(\mathrm{V}_{1} \mathrm{~V}_{\mathrm{o}}\right)$ and this holoenzyme in complex with a bacterial effector $\left(\mathrm{V}_{1} \mathrm{~V}_{\mathrm{o}}\right.$-VopQ). Using high resolution structures containing bound nucleotides (AMPPNP and ADP), we illustrate the mechanism of enzyme catalysis in a V-ATPase holoenzyme complex.

\section{Results}

\section{Structure determination of the intact yeast V-ATPase holoenzyme}

Yeast V-ATPase contains two isoforms of subunit a (Vph1p and Stv1p) and only one isoform of all the other subunits ${ }^{6,18,19}$. Therefore, to obtain a homogeneous yeast V-ATPase complex with the single subunit a isoform (Vph1p), we deleted Stv1p. This complex was supplemented with an 
ATP analog, AMP-PNP, in order to capture different conformations of V-ATPase and stabilize the complex by using this hydrolysis-deficient substrate. Cryo-EM data analysis indicates the presence of state 1 and state 2 complexes, but not state 3 , with the majority of particles in state 1 (Extended Data Fig. 1). We propose that state 3 likely disassembles during the purification steps, consistent with the V-ATPase disassembly model that state 3 is where disassembly starts ${ }^{15,20,21}$. The $\mathrm{V}_{1} \mathrm{~V}_{\mathrm{o}}$ complex obtained was intact with subunit $\mathrm{H}$ density maps resolved. State 1 of the $V_{1} V_{0}$ complex was determined at a reported overall resolution of $3.14 \AA$, with focused refinements further improving local resolutions (Extended Data Fig. 1,3,4). State 2 was resolved at reported overall resolution of $3.53 \AA$, however, the local density maps were worse due to the problem of preferred orientation (Extended Data Fig. 3d). The yeast $V_{1} V_{o}$ protein sample was supplemented with AMP-PNP causing closure of the catalytic subunit pair of $\mathrm{A}_{(1)}-\mathrm{B}_{(1)}$ (Fig. 1). A gray line in the model is drawn through the A-B subunit pairs to draw attention their open and closed states. Previously, this same pair was shown to be in a loose conformation in nucleotide free environment ${ }^{15}$. The catalytic subunit pair of $\mathrm{A}_{(2)}-\mathrm{B}_{(2)}$ are in an open conformation. The third pair, $\mathrm{A}_{(3)}-\mathrm{B}_{(3)}$ is in a closed state and occupied by an AMP-PNP molecule that replaces ADP as was modelled in the rat V-ATPase ${ }^{8}$.

\section{$V_{1} V_{0}$ holoenzyme in complex with a bacterial effector}

A Vibrio parahaemolyticus type 3 secretion system effector, VopQ, was previously shown to bind dissociated $V_{o}$ subcomplex and predicted to bind the holoenzyme of $V_{1} V_{o}$ in state 2 (ref. ${ }^{17}$ ). To gain better insights into $\mathrm{V}_{1} \mathrm{~V}_{\mathrm{o}}$ and test if $\mathrm{VopQ}$ could indeed interact with the holoenzyme, we prepared the $\mathrm{V}_{1} \mathrm{~V}_{\mathrm{o}}$ sample with VopQ present and analyzed the cryo-EM data (Extended Data Fig. 2,3). Both AMP-PNP and ADP were supplemented in the $V_{1} V_{0}-V o p Q$ sample to obtain 
ADP bound V-ATPase. As previously predicted, $V_{1} V_{0^{-}}-V_{0 p Q}$ complex was found to be exclusively in state 2 and the angular distribution was restored to 3 major preferred orientations, similar to the $\mathrm{V}_{1} \mathrm{~V}_{\mathrm{o}}$ complex in state 1 (Extended Data Fig. 3d). This observation suggests that VopQ interacts with $\mathrm{V}_{1} \mathrm{~V}_{\mathrm{o}}$ in state 2 and locks the enzyme in this state. The final 3D reconstruction improved the cryo-EM density map quality and allowed characterization of $\mathrm{V}_{1} \mathrm{~V}_{\mathrm{o}}$ in state 2 (Extended Data Fig. 4).

A detailed examination and comparison of the 3D class cryo-EM density maps indicated that VopQ was bound with $\mathrm{V}_{1} \mathrm{~V}_{\mathrm{o}}$ in state 2 as predicted (Extended Data Fig. 5a). However, the density maps for VopQ were not well resolved, indicating that VopQ may partially clashed with subunits in $\mathrm{V}_{1} \mathrm{~V}_{\mathrm{o}}$ and the relatively dynamic $\mathrm{V}_{\mathrm{o}}$ regions may also contribute to this clash. A VopQ molecule corresponding to VopQ-2B in the reported $V_{0^{-}}-$VopQ structures ${ }^{17}$ was docked into the cryo-EM density map (Fig. 2a). To further support the hypothesis that VopQ interacts with $\mathrm{V}_{1} \mathrm{~V}_{\mathrm{o}}$ in state 2, we performed an ATPase activity inhibition assay (Extended Data Fig. 5b). VopQ showed no inhibition on ATP hydrolysis when ATP was added before $V_{1} V_{\text {o. }}$ But when VopQ was pre-incubated with $\mathrm{V}_{1} \mathrm{~V}_{\mathrm{o}}$ before ATP was added, a partial inhibition of ATP hydrolysis was observed. This result suggests that the interaction between VopQ and $V_{1} V_{o}$ requires $\mathrm{V}_{1} \mathrm{~V}_{\mathrm{o}}$ to be relatively stable in state 2 so VopQ can bind while changing from a soluble state to a membrane imbedded and $\mathrm{V}_{1} \mathrm{~V}_{\mathrm{o}}$-bound state. Under both conditions, the V-ATPase inhibitor folimycin ${ }^{22}$ could completely block ATP hydrolysis, implying a rapid binding of this inhibitor. The complete inhibition by folimycin indicates a tight coupling between $\mathrm{V}_{1}$ ATP hydrolysis and $\mathrm{V}_{\mathrm{o}}$ proton transport as it works at the transmembrane region of $\mathrm{V}_{\mathrm{o}}$ subcomplex ${ }^{23}$. Disassembled $\mathrm{V}_{1}$ will continuously drive ATP hydrolysis without subunit $\mathrm{H}$ which plays an inhibitory role ${ }^{24}$. This activity will not be inhibited by $\mathrm{V}_{\mathrm{o}}$ inhibitors (folimycin or 
bafilomycin $)^{23}$ as was observed for the rat V-ATPase that lacks subunit $\mathrm{H}^{8}$. Therefore, our data also suggests that subunit $\mathrm{H}$ is present in possible disassembled $\mathrm{V}_{1}$ subcomplex and in the holocomplex, supported by the fact that subunit $\mathrm{H}$ is essential for the holoenzyme activity ${ }^{25}$. (closed), $\mathrm{A}_{(1)}-\mathrm{B}_{(1)}$ (open), and $\mathrm{A}_{(2)}-\mathrm{B}_{(2)}$ (closed) correspond to those of $\mathrm{A}_{(1)}-\mathrm{B}_{(1)}, \mathrm{A}_{(2)}-\mathrm{B}_{(2)}$, and $A_{(3)}-B_{(3)}$ in state 1 due to enzyme reaction and rotation of rotor subunits (Fig. 2a). The catalytic site of the $\mathrm{A}_{(2)}-\mathrm{B}_{(2)}$ pair is bound with an ADP molecule rather than an AMP-PNP molecule. The overall conformations of catalytic subunit pairs are similar to those in the previously published disassembled $\mathrm{V}_{1}$ (not shown), which is in an inhibited state similar to state 2 (ref. ${ }^{26}$ ).

\section{Interaction of subunit $H$ with the other V-ATPase subunits}

Subunit $\mathrm{H}$ is a regulator that inhibits ATP hydrolysis by disassembled $\mathrm{V}_{1}$ and interestingly,

subunit $\mathrm{H}$ is necessary for the activity of the holoenzyme ${ }^{24,25,27}$. In the yeast holoenzyme obtained above, although subunit $\mathrm{H}$ was present, the intra-complex dynamics between $\mathrm{V}_{1}$ and $\mathrm{V}_{\mathrm{o}}$ subcomplexes hindered analysis of the interaction between subunit $\mathrm{H}$ and neighboring subunits. The $\mathrm{V}_{1} \mathrm{~V}_{\mathrm{o}}$-VopQ complex displayed better map quality for subunit $\mathrm{H}$ and allowed definition of this interaction, possibly stabilized by the binding of the protein inhibitor VopQ (Extended Data Fig. 4).

The N-terminal domain of subunit $\mathrm{H}$ is in close contact with $\mathrm{V}_{1}$ subunit $\mathrm{E}_{(1)}$, subunit $\mathrm{G}_{(1)}$, and $\mathrm{V}_{\mathrm{o}}$ subunit a, while the $\mathrm{C}$-terminal domain is contacting $\mathrm{V}_{\mathrm{o}}$ subunit a but not the $\mathrm{V}_{1}$ subcomplex (Fig. 2a). Density-supported interaction pairs include (* indicates main chain atom): Y244-E30_E(1), Y244-E30*_E(1), F255*-Q41_E(1), K283-E27_E(1), K283-E24_a, and K329E58_a (N-terminal, Fig. 2b); Y463-Q123_a and Q470-Q123_a (C-terminal, Fig. 2c). These 
interactions are in contrast to the yeast $\mathrm{V}$-ATPase $\mathrm{V}_{1}$ subcomplex where the $\mathrm{C}$-terminal domain is folded up toward the $\mathrm{V}_{1}$ subunits $\mathrm{B}_{(1)}$ and $\mathrm{D}^{26}$. Our structures support the previous model

\section{Conformations of the catalytic subunit pairs}

As mentioned above, $\mathrm{A}_{(1)}-\mathrm{B}_{(1)}, \mathrm{A}_{(2)}-\mathrm{B}_{(2)}$, and $\mathrm{A}_{(3)}-\mathrm{B}_{(3)}$ in state 1 are in closed, open, and closed conformations. The $\mathrm{A}_{(1)}-\mathrm{B}_{(1)}$ in state 1 is captured in a closed conformation due to the binding of non-hydrolysable AMP-PNP (Fig. 3a). The natural product of ATP hydrolyses, ADP, is modelled in the rat $\mathrm{V}$-ATPase ${ }^{8} \mathrm{~A}_{(3)}-\mathrm{B}_{(3)}$ pair and is replaced in this structure by AMP-PNP, fitting well in the density map (Extended Data Fig. 4c). In the $\mathrm{V}_{1} \mathrm{~V}_{\mathrm{o}}-\mathrm{VopQ}$ complex in state 2, similar conformational arrangement is observed for $\mathrm{A}_{(1)}-\mathrm{B}_{(1)}, \mathrm{A}_{(2)}-\mathrm{B}_{(2)}$, and $\mathrm{A}_{(3)}-\mathrm{B}_{(3)}$ with pairs in the open, closed, and closed conformations, respectively. However, the $\mathrm{A}_{(2)}-\mathrm{B}_{(2)}$ catalytic site for the $\mathrm{V}_{1} \mathrm{~V}_{\mathrm{o}^{-}}$-VopQ complex is occupied by an ADP molecule instead of AMP-PNP (Fig. 3b;

Extended Data Fig. 4c). As both ADP and AMP-PNP were supplemented at equal concentration to the final sample, this might support the proposal that ADP has a higher affinity at this site than AMP-PNP. Conversely, AMP-PNP might have a higher affinity at the $\mathrm{A}_{(3)}-\mathrm{B}_{(3)}$ catalytic site. The two catalytic sites in the $\mathrm{V}_{1} \mathrm{~V}_{\mathrm{o}}$ in state 2 without VopQ, where ADP is not 
provided, are both occupied by AMP-PNP supported by the density maps (Extended Data Fig. 4c).

V-ATPase is a member of rotary ATPases that also include F-ATPase and A-ATPase that can both synthesize and hydrolyze $\mathrm{ATP}^{11-13}$. Rotary ATPase are dynamic protein machines that have been captured in many states and conformations, either for the subcomplexes or the holoenzymes. Rotary ATPases share similarities in architectures and working mechanisms (Extended Data Fig. 6a). When visualizing the catalytic subunits from the membrane side in representative rotary ATPase structures and placing the short N-terminal helix of subunit $\gamma$ in F-

(Extended Data Fig. 6b-6e), the top catalytic pairs are in open and therefore empty conformations. This regular pattern also applies to our structures (Fig. 3a,3b). By comparing our structures with and a bacterial intact V/A-ATPase and other eukaryotic V-ATPase structures $\left(\mathrm{V}_{1}\right.$ or $\mathrm{V}_{1} \mathrm{~V}_{\mathrm{o}}$ ) based on this pattern, it is obvious that yeast V-ATPase structures are in different states with two of the three catalytic A-B pairs occupied by nucleotides ${ }^{8,15,16}$ (Fig. 3a,3b; Extended Data Fig. 6d,6e).

V-ATPase catalytic subunit (A or B) can be roughly divided into three domains, an Nterminal $\beta$-barrel domain, a central nucleotide-binding domain (for subunit $\mathrm{A}$, an additional nonhomologous domain), and a C-terminal domain ${ }^{29}$ (Extended Data Fig. 7a). The conformations of each catalytic subunit in $\mathrm{V}_{1} \mathrm{~V}_{\mathrm{o}}$ (state 1) is examined and compared (Extended Data Fig. 7b). Subunit $A$ and $B$ in $A_{(1)}-B_{(1)}$ pair are almost identical to those in $A_{(3)}-B_{(3)}$ pair, resulting in small rigid movement of the $\mathrm{A}_{(1)}-\mathrm{B}_{(1)}$ pair compared to $\mathrm{A}_{(3)}-\mathrm{B}_{(3)}$ when aligned against the N-terminal $\beta$ barrel domains (Fig. 3c). Subunit A or B in $\mathrm{A}_{(2)}-\mathrm{B}_{(2)}$ pair undergoes obvious intra-subunit conformations changes, contributing to their open conformation (Fig. 3c; Extended Data Fig. 
7b). In comparison with the rat V-ATPase in state 1 , the yeast $\mathrm{A}_{(1)}-\mathrm{B}_{(1)}$ pair shows an obvious more closed conformation while the other two pairs are more or less similar (Fig. 3c; Extended Data Fig. 7c). The $A_{(3)}-B_{(3)}, A_{(1)}-B_{(1)}$, and $A_{(2)}-B_{(2)}$ pairs in $V_{1} V_{0}-V o p Q$ of state 2 are in similar intra-subunit and subunit-pair conformations with $\mathrm{A}_{(1)}-\mathrm{B}_{(1)}, \mathrm{A}_{(2)}-\mathrm{B}_{(2)}$, and $\mathrm{A}_{(3)}-\mathrm{B}_{(3)}$ pairs in $\mathrm{V}_{1} \mathrm{~V}_{\mathrm{o}}$ of state 1 (Extended Data Fig. 8). These observed conformations represent pre-catalysis states of the catalytic subunits in the holoenzyme, captured using non-hydrolysable AMP-PNP.

\section{Discussion}

In the yeast V-ATPase structures described above, extended residues of subunit $\mathrm{B}$ ( $\mathrm{N}$ terminus) and subunits $\mathrm{E}$ and $\mathrm{G}$ (C terminus) were modelled compared to the previous low-resolution structures ${ }^{15}$. These residues indicate additional interaction interfaces between subunit B, E, and $\mathrm{G}$ in the yeast V-ATPase as described for the rat V-ATPase ${ }^{8}$. The bacterial effector VopQ is capable of binding the disassembled $\mathrm{V}_{\mathrm{o}}$ subcomplex ${ }^{17}$. As in silico predicted, VopQ helps to resolve the V-ATPase structure in state 2 as a state-specific inhibitor, making it a useful tool for future V-ATPase studies.

Eukaryotic V-ATPase is a member of rotary ATPases that differs from F-ATPase and AATPase. With the high-resolution intact yeast V-ATPase structures, we are able to visualize the proton pump with subunit $\mathrm{H}$ assembled which is an important regulator of eukaryotic V-ATPase. Studies suggest that subunit $\mathrm{H}$ prevents energy waste of ATP hydrolysis in disassembled $\mathrm{V}_{1}$ subcomplex ${ }^{24,26}$. In the V-ATPase holoenzyme, it is necessary for activity but not assembly ${ }^{25,27}$. Since subunit $H$ only contacts $V_{1}$ subunits $E_{(1)}$ and $G_{(1)}$ and $V_{o}$ subunit a in the holoenzyme, it is likely that subunit $\mathrm{H}$ holds the framework of stator subunits and allows ATP hydrolysis by the catalytic subunits. Though the reported rat V-ATPase structures without subunit $\mathrm{H}^{8}$ show 
differences in the stator subunits compared to our yeast structures (not shown), it is not known if these differences result from sample variety. Therefore, systematic studies of a eukaryotic VATPase from a single organism is necessary for revealing possibly subtle differences between different states or conformations, which will contribute to an understanding of subunit $\mathrm{H}$ function. This calls for structures of yeast V-ATPase without subunit $\mathrm{H}$ or structures of rat VATPase with subunit $\mathrm{H}$. The yeast V-ATPase represents a better option as extensive studies have been performed on it and it is easier to manipulate the much less genes of subunit isoforms in yeast for biophysical and biochemical tests.

Catalysis mechanisms for rotary ATPases have been proposed mostly based on $F_{1}$ and $V_{1}$ subcomplexes ${ }^{13,14,29}$. However, these subcomplexes only contain the core catalytic subunits and rotor subunits, with some even lacking the rotor subunits ${ }^{29}$. The incompleteness of these subcomplexes will cause structure feature changes compared to the holoenzymes as observed $^{14,29}$. These changes are likely artificial. Even the disassembled intact yeast $\mathrm{V}_{1}$ subcomplex, which is physiologically related, is locked in an auto-inhibited conformation that differs from the holoenzyme ${ }^{26}$. Therefore, direct observation of various conformations of catalytic subunit pairs in a V-ATPase holoenzyme was needed to test the rotary catalysis mechanism. By determining the conformations of catalytic subunit pairs in the yeast V-ATPase holoenzyme, we revealed two similar nucleotide-bound conformations and one open conformation. These observations in a V-ATPase holoenzyme support the previous model ${ }^{13,14,30}$ of a rotary catalysis mechanism wherein: (1) two catalytic subunit pairs bind ATP molecules with one being empty; (2) the ATP bound pair after the empty in the rotation direction catalyzes ATP hydrolysis; (3) ADP and phosphate get released and the empty pair is occupied by an ATP molecule; (4) the catalytic subunit pairs repeat the above steps. Therefore, our studies provide a 
bioRxiv preprint doi: https://doi.org/10.1101/2020.07.16.207225; this version posted July 17, 2020. The copyright holder for this preprint (which was not certified by peer review) is the author/funder. All rights reserved. No reuse allowed without permission.

222 mechanistic insight into a fully assembled and functional V-ATPase and are helpful for future 223 studies to address other rotary ATPases. 


\section{Acknowledgments}

We thank X. Li and the Orth lab members for discussions and editing. We thank the Friedman laboratory for assistance with yeast cell disruption. We thank the Structural Biology Laboratory (SBL) for assistance with cryo-EM grid preparation and screening. Cryo-EM data were collected at the University of Texas Southwestern Medical Center Cryo-Electron Microscopy Facility that is funded in part by the CPRIT Core Facility Support Award RP170644. We thank SBL for providing computational resources for cryo-EM data processing. This work was funded by the Welch Foundation grant I-1561 (K.O.), Once Upon a Time...Foundation (K.O.), and National Institutes of Health Grant R01 GM115188 (K.O.). K.O. is a W.W. Caruth, Jr. Biomedical

\section{Author contributions}

W.P. and K.O. conceived the project and designed the experiments. W.P., J.F., A.K.C., and authors. K.O. supervised the project.

\section{Competing interests}

The authors declare no competing interests. 
Methods

\section{Purification of the Yeast V-ATPase Complex}

The S. cerevisiae TAP-tagged VMA1 strain (YSC1178-202230289, Dharmacon, Inc.) was purchased and the TAP tag was replaced by a $3 \times$ FLAG tag after $S T V 1$ was knocked out ${ }^{31}$. Yeast cells (8 9 liters in flasks) were cultured to logarithmic phase which helped maintain an intact holoenzyme because of abundant glucose ${ }^{32}$. Collected cells were resuspended in lysis buffer (25 glucose, $8 \%$ sucrose, $2 \%$ sorbitol) and frozen in liquid nitrogen as dropped beads (diameter less than $3 \mathrm{~mm}$ ). The frozen cells were disrupted using a 6870D Freezer/Mill ${ }^{\circledR}$ Dual Chamber Cryogenic Grinder (SPEX ${ }^{\mathrm{TM}}$ SamplePrep). The powder thawed and the cell debris was removed by low-speed centrifugation (1,250 g, $10 \mathrm{~min})$. V-ATPase containing membrane was collected by high-speed centrifugation $(27,000 \mathrm{~g}, 1 \mathrm{~h})$. The membrane was solubilized with detergent mixture (2\% n-Dodecyl- $\beta$-D-Maltoside (DDM), 0.5\% 3-((3-Cholamidopropyl) Dimethylammonio)-1-Propanesulfonate (CHAPS), 0.05\% Cholesteryl Hemisuccinate Tris Salt (CHS)). Insoluble materials were removed by high-speed centrifugation. The supernatant was loaded into gravity column containing anti-FLAG M2 affinity gel (GE Healthcare). The resin was washed with wash buffer (25 mM HEPES pH 7.4, $150 \mathrm{mM} \mathrm{NaCl,} 1 \mathrm{mM}$ PMSF, protease inhibitor cocktail, $0.05 \%$ glyco-diosgenin (GDN)). Bound protein was eluted with elution buffer (25 mM HEPES pH 7.4, $150 \mathrm{mM} \mathrm{NaCl,} 1 \mathrm{mM}$ PMSF, protease inhibitor cocktail, 0.05\% GDN, $100 \mu \mathrm{g} / \mathrm{mL} 3 \times$ FLAG peptide (Sigma)). The eluted protein sample was concentrated and applied to gel filtration (Superose 6 10/300 GL, GE Healthcare) in running buffer (25 mM HEPES pH 7.4, $150 \mathrm{mM} \mathrm{NaCl}, 2 \mathrm{mM}$ DTT, 0.05\% GDN). Fractions containing the V-ATPase complexes were pooled together and concentrated to around $40 \mu \mathrm{L}$ (less than $2 \mathrm{mg} / \mathrm{mL}$ ). 
For preparation of $\mathrm{V}_{1} \mathrm{~V}_{\mathrm{o}}$-VopQ complex, VopQ protein was obtained as previously described $^{17} .0 .9 \mathrm{mg}$ of VopQ protein was added at the membrane solubilization step. $0.2 \mathrm{mg}$ of VopQ protein was added again after the complex eluted from the anti-FLAG column. The detergent used after membrane solubilization was $0.05 \%$ digitonin instead of GDN.

For cryo-EM sample preparation, $2 \mathrm{mM} \mathrm{MgCl}_{2}$ and $0.2 \mathrm{mM}$ AMP-PNP were added into the $\mathrm{V}_{1} \mathrm{~V}_{\mathrm{o}}$ sample. $2 \mathrm{mM} \mathrm{MgCl}$, $0.2 \mathrm{mM}$ AMP-PNP, and $0.2 \mathrm{mM}$ ADP were added into the

\section{Malachite Green Assay}

Malachite green assay was performed as described with some modifications ${ }^{33}$. Briefly, malachite green oxalate $(13.5 \mathrm{mg})$ was dissolved in $30 \mathrm{~mL}$ of Milli-Q $\mathrm{H}_{2} \mathrm{O} .10 \mathrm{~mL}$ of $4.2 \%$ (w/v)

ammonium molybdate in $4 \mathrm{M} \mathrm{HCl}$ was then added. This solution was incubated on a nutator at 4 ${ }^{\circ} \mathrm{C}$ for at least 45 minutes. The solution was filtered and $0.01 \%$ Tween 20 was added before use. The malachite green assay buffer contained 25 mM HEPES (pH 7.4), 150 mM NaCl, 2 $\mathrm{mM} \mathrm{MgCl}$, and $0.02 \%$ DDM. To start the assay, $0.005 \mu \mathrm{M} \mathrm{V}_{1} \mathrm{~V}_{\mathrm{o}}$ and $0.3 \mathrm{mM}$ ATP was incubated at room temperature $\left(\sim 25^{\circ} \mathrm{C}\right)$ for $10 \mathrm{~min}$ with a volume of $100 \mu \mathrm{L}$. VopQ $(1 \mu \mathrm{M})$ and the V-ATPase inhibitor folimycin $(1 \mu \mathrm{M})$ were tested for ATPase activity inhibition. Reactions were stopped by adding $800 \mu \mathrm{L}$ malachite green reagent followed by addition of $100 \mu \mathrm{L} 34 \%$ $(\mathrm{w} / \mathrm{v})$ sodium citrate. The solutions were measured for absorbance at $620 \mathrm{~nm}$ in technical 


\section{Cryo-EM Image Collection}

Cryo-EM grids were prepared using Vitrobot Mark IV (Thermo Fisher Scientific). Quantifoil R1.2/1.3 300-mesh gold holey carbon grids were coated with carbon film with thickness of 1 3 nm (Compact Coating Unit CCU-010, Safematic). $3.5 \mu \mathrm{L}$ of protein sample was placed on a glow-discharged grid. After a waiting time of $15 \mathrm{~s}$, the grid was blotted for $1.5 \mathrm{~s}$ under $100 \%$ humidity at $8^{\circ} \mathrm{C}$ before plunged into liquid ethane. For grid screening, micrographs were acquired on a Talos Arctica microscope (Thermo Fisher Scientific) operated at $200 \mathrm{kV}$ with a K3 direct electron detector (Gatan). The micrographs were recorded with SerialEM ${ }^{34}$. For data collection, micrographs were acquired on a Titan Krios microscope (Thermo Fisher Scientific) operated at $300 \mathrm{kV}$ with a $\mathrm{K} 3$ direct electron detector, using a slit width of $20 \mathrm{eV}$ on a GIFQuantum energy filter. Images were recorded using SerialEM at a calibrated magnification of 59,983 with a super-resolution pixel size of $0.415 \AA$. The defocus range was set from $-1.0 \mu \mathrm{m}$ to $-2.5 \mu \mathrm{m}$. Each micrograph was dose-fractionated $(0.05 \mathrm{~s} /$ frame $)$ with a total dose of around $60 \mathrm{e}^{-}$ $/ \AA^{2}$.

\section{Cryo-EM Image Processing}

Image processing was done roughly following the RELION-3.0 and RELION-3.1 user manuals ${ }^{35}$. The Titan Krios micrographs were motion corrected and binned two-fold with MotionCor2 (ref. ${ }^{36}$ ), yielding a pixel size of $0.83 \AA$ A. The CTF parameters of the micrographs were estimated using CTFFIND 4 (ref. ${ }^{37}$ ). All other steps of image processing were performed using RELION ${ }^{35}$. Around 1,000 particles were manually picked from a few micrographs for initial 2D classification and template generation for auto-picking. Particles were then autopicked from selected micrographs. After subgroup 2D and 3D classification with a binning factor of 2 or 
4 for fast calculation, good class particles were selected for further processing. 3D classification with a binning factor of 1 allowed further separation of good particles from the bad and classification of different states. CTF-refinement was performed to improve the resolution of 3D reconstruction. Multi-body refinement was performed to analyze local regions with better map quality. The resolutions were estimated according to the gold-standard Fourier shell correlation (FSC) 0.143 criterion. RELION was used to calculate the local resolution map ${ }^{35}$. Detailed workflows of image processing and statistics are shown in Extended Data Figs. 1-3 and Extended Data Table 1.

\section{Model Building and Structure Refinement}

The structures of yeast $\mathrm{V}_{1} \mathrm{~V}_{\mathrm{o}}(\mathrm{PDB} \text { code: } 3 \mathrm{~J} 9 \mathrm{U})^{7}$ and $\mathrm{V}_{\mathrm{o}^{-}} \mathrm{VopQ}(\mathrm{PDB} \text { code: } 6 \mathrm{PE} 5)^{17}$ were used as initial models for model building of $\mathrm{V}_{1} \mathrm{~V}_{\mathrm{o}}$-VopQ (state2). The model was manually adjusted in $\mathrm{COOT}^{38}$. The structure of $\mathrm{V}_{1} \mathrm{~V}_{\mathrm{o}}-\mathrm{VopQ}$ was then used as initial model for $\mathrm{V}_{1} \mathrm{~V}_{\mathrm{o}}$ (state 1 and state 2). Structure refinement was performed using PHENIX ${ }^{39}$ in real space with secondary structure and geometry restrained. The statistics of the geometries of the models were generated using MolProbity ${ }^{40}$. The $\mathrm{V}_{1} \mathrm{~V}_{\mathrm{o}^{-}}$-VopQ model was validated without VopQ as the density for VopQ was not well resolved. Multibody refinement maps were merged in UCSF CHIMERA ${ }^{41}$ to generate a single map for validation of the overall models. 


\section{Data Availability}

339 The atomic coordinates of the yeast V-ATPase complexes are deposited in the Protein Data Bank 340 with accession codes $6 \mathrm{XS} 0\left(\mathrm{~V}_{1} \mathrm{~V}_{\mathrm{o}}\right.$ state 1$), 6 \mathrm{XS1}\left(\mathrm{V}_{1} \mathrm{~V}_{\mathrm{o}}\right.$ state 2$)$, and $6 \mathrm{XS} 2\left(\mathrm{~V}_{1} \mathrm{~V}_{\mathrm{o}}-\mathrm{VopQ}\right.$ state 2$)$. 341 The corresponding electron microscopy density maps are deposited in the Electron Microscopy 342 Data Bank with accession codes EMD-22298 ( $\mathrm{V}_{1} \mathrm{~V}_{\mathrm{o}}$ state 1), EMD-22299 ( $\mathrm{V}_{1} \mathrm{~V}_{\mathrm{o}}$ state 2$)$, and 343 EMD-22300 ( $\mathrm{V}_{1} \mathrm{~V}_{\mathrm{o}}$-VopQ state 2$)$. 


\begin{tabular}{|c|c|c|}
\hline \multirow{2}{*}{$\begin{array}{l}346 \\
347\end{array}$} & \multicolumn{2}{|c|}{ References } \\
\hline & & \\
\hline 348 & 1. & Forgac, M. Vacuolar ATPases: rotary proton pumps in physiology and pathophysiology. \\
\hline 349 & & Nat Rev Mol Cell Biol 8, 917-29 (2007). \\
\hline 350 & 2. & Sun-Wada, G.H. \& Wada, Y. Role of vacuolar-type proton ATPase in signal \\
\hline 351 & & transduction. Biochim Biophys Acta 1847, 1166-72 (2015). \\
\hline 352 & 3. & Qin, A. et al. V-ATPases in osteoclasts: structure, function and potential inhibitors of \\
\hline 353 & & bone resorption. Int J Biochem Cell Biol 44, 1422-35 (2012). \\
\hline 354 & 4. & Vavassori, S. \& Mayer, A. A new life for an old pump: V-ATPase and neurotransmitter \\
\hline 355 & & release. J Cell Biol 205, 7-9 (2014). \\
\hline 356 & 5. & Breton, S. \& Brown, D. Regulation of luminal acidification by the V-ATPase. Physiology \\
\hline 357 & & (Bethesda) 28, 318-29 (2013). \\
\hline 358 & 6. & Toei, M., Saum, R. \& Forgac, M. Regulation and isoform function of the V-ATPases. \\
\hline 359 & & Biochemistry 49, 4715-23 (2010). \\
\hline 360 & 7. & Roh, S.H. et al. The 3.5-A CryoEM Structure of Nanodisc-Reconstituted Yeast Vacuolar \\
\hline 361 & & ATPase Vo Proton Channel. Mol Cell 69, 993-1004 e3 (2018). \\
\hline 362 & 8. & Abbas, Y.M., Wu, D., Bueler, S.A., Robinson, C.V. \& Rubinstein, J.L. Structure of V- \\
\hline 363 & & ATPase from the mammalian brain. Science $367,1240-1246$ (2020). \\
\hline 364 & 9. & Cotter, K., Stransky, L., McGuire, C. \& Forgac, M. Recent Insights into the Structure, \\
\hline 365 & & Regulation, and Function of the V-ATPases. Trends Biochem Sci 40, 611-622 (2015). \\
\hline 366 & 10. & Vasanthakumar, T. \& Rubinstein, J.L. Structure and Roles of V-type ATPases. Trends \\
\hline 367 & & Biochem Sci 45, 295-307 (2020). \\
\hline 368 & 11. & Kuhlbrandt, W. \& Davies, K.M. Rotary ATPases: A New Twist to an Ancient Machine. \\
\hline 369 & & Trends Biochem Sci 41, 106-116 (2016). \\
\hline 370 & 12. & Kuhlbrandt, W. Structure and Mechanisms of F-Type ATP Synthases. Annu Rev Biochem \\
\hline 371 & & $88,515-549(2019)$ \\
\hline 372 & 13. & Walker, J.E. The ATP synthase: the understood, the uncertain and the unknown. Biochem \\
\hline 373 & & Soc Trans 41, 1-16 (2013). \\
\hline 374 & 14. & Arai, S. et al. Rotation mechanism of Enterococcus hirae V1-ATPase based on \\
\hline 375 & & asymmetric crystal structures. Nature 493, 703-7 (2013). \\
\hline 376 & 15. & Zhao, J., Benlekbir, S. \& Rubinstein, J.L. Electron cryomicroscopy observation of \\
\hline 377 & & rotational states in a eukaryotic V-ATPase. Nature 521, 241-5 (2015). \\
\hline 378 & 16. & Zhou, L. \& Sazanov, L.A. Structure and conformational plasticity of the intact Thermus \\
\hline 379 & & thermophilus V/A-type ATPase. Science 365(2019). \\
\hline 380 & 17. & Peng, W. et al. A distinct inhibitory mechanism of the V-ATPase by Vibrio VopQ \\
\hline 381 & & revealed by cryo-EM. Nat Struct Mol Biol 27, 589-597 (2020). \\
\hline 382 & 18. & Manolson, M.F. et al. The VPH1 gene encodes a 95-kDa integral membrane polypeptide \\
\hline 383 & & required for in vivo assembly and activity of the yeast vacuolar $\mathrm{H}(+)$-ATPase. $J$ Biol \\
\hline 384 & & Chem 267, 14294-303 (1992). \\
\hline 385 & 19. & Manolson, M.F. et al. STV1 gene encodes functional homologue of 95-kDa yeast \\
\hline 386 & & vacuolar H(+)-ATPase subunit Vph1p. J Biol Chem 269, 14064-74 (1994). \\
\hline 387 & 20. & Mazhab-Jafari, M.T. et al. Atomic model for the membrane-embedded VO motor of a \\
\hline 388 & & eukaryotic V-ATPase. Nature 539, 118-122 (2016). \\
\hline 389 & 21. & Oot, R.A., Couoh-Cardel, S., Sharma, S., Stam, N.J. \& Wilkens, S. Breaking up and \\
\hline 390 & & making up: The secret life of the vacuolar H(+) -ATPase. Protein Sci 26, 896-909 (2017 \\
\hline
\end{tabular}


22. Muroi, M., Shiragami, N., Nagao, K., Yamasaki, M. \& Takatsuki, A. Folimycin (concanamycin A), a specific inhibitor of V-ATPase, blocks intracellular translocation of the glycoprotein of vesicular stomatitis virus before arrival to the Golgi apparatus. Cell Struct Funct 18, 139-49 (1993).

23. Bowman, B.J., McCall, M.E., Baertsch, R. \& Bowman, E.J. A model for the proteolipid ring and bafilomycin/concanamycin-binding site in the vacuolar ATPase of Neurospora crassa. J Biol Chem 281, 31885-93 (2006).

24. Parra, K.J., Keenan, K.L. \& Kane, P.M. The H subunit (Vma13p) of the yeast V-ATPase inhibits the ATPase activity of cytosolic V1 complexes. J Biol Chem 275, 21761-7 (2000).

25. Ho, M.N. et al. VMA13 encodes a 54-kDa vacuolar H(+)-ATPase subunit required for activity but not assembly of the enzyme complex in Saccharomyces cerevisiae. J Biol Chem 268, 18286-92 (1993).

26. Oot, R.A., Kane, P.M., Berry, E.A. \& Wilkens, S. Crystal structure of yeast V1-ATPase in the autoinhibited state. EMBO J 35, 1694-706 (2016).

27. Crider, B.P. \& Xie, X.S. Characterization of the functional coupling of bovine brain vacuolar-type $\mathrm{H}(+)$-translocating ATPase. Effect of divalent cations, phospholipids, and subunit H (SFD). J Biol Chem 278, 44281-8 (2003).

28. Dingerdissen, H.M. et al. BioMuta and BioXpress: mutation and expression knowledgebases for cancer biomarker discovery. Nucleic Acids Res 46, D1128-D1136 (2018).

29. Maher, M.J. et al. Crystal structure of A3B3 complex of V-ATPase from Thermus thermophilus. EMBO J 28, 3771-9 (2009).

30. Menz, R.I., Walker, J.E. \& Leslie, A.G. Structure of bovine mitochondrial F(1)-ATPase with nucleotide bound to all three catalytic sites: implications for the mechanism of rotary catalysis. Cell 106, 331-41 (2001).

31. Longtine, M.S. et al. Additional modules for versatile and economical PCR-based gene deletion and modification in Saccharomyces cerevisiae. Yeast 14, 953-61 (1998).

32. Kane, P.M. Disassembly and reassembly of the yeast vacuolar H(+)-ATPase in vivo. $J$ Biol Chem 270, 17025-32 (1995).

33. Lopez, V.A. et al. A Bacterial Effector Mimics a Host HSP90 Client to Undermine Immunity. Cell 179, 205-218 e21 (2019).

34. Mastronarde, D.N. Automated electron microscope tomography using robust prediction of specimen movements. J Struct Biol 152, 36-51 (2005).

35. Zivanov, J. et al. New tools for automated high-resolution cryo-EM structure determination in RELION-3. Elife 7(2018).

36. Zheng, S.Q. et al. MotionCor2: anisotropic correction of beam-induced motion for improved cryo-electron microscopy. Nat Methods 14, 331-332 (2017).

37. Rohou, A. \& Grigorieff, N. CTFFIND4: Fast and accurate defocus estimation from electron micrographs. J Struct Biol 192, 216-21 (2015).

38. Emsley, P., Lohkamp, B., Scott, W.G. \& Cowtan, K. Features and development of Coot. Acta Crystallogr D Biol Crystallogr 66, 486-501 (2010).

39. Adams, P.D. et al. PHENIX: a comprehensive Python-based system for macromolecular structure solution. Acta Crystallogr D Biol Crystallogr 66, 213-21 (2010).

40. Chen, V.B. et al. MolProbity: all-atom structure validation for macromolecular crystallography. Acta Crystallogr D Biol Crystallogr 66, 12-21 (2010). 
41. Pettersen, E.F. et al. UCSF Chimera--a visualization system for exploratory research and

42. Goddard, T.D. et al. UCSF ChimeraX: Meeting modern challenges in visualization and analysis. Protein Sci 27, 14-25 (2018).

43. Schrödinger, L. The PyMOL Molecular Graphics System, Version 1.8. (2015). 


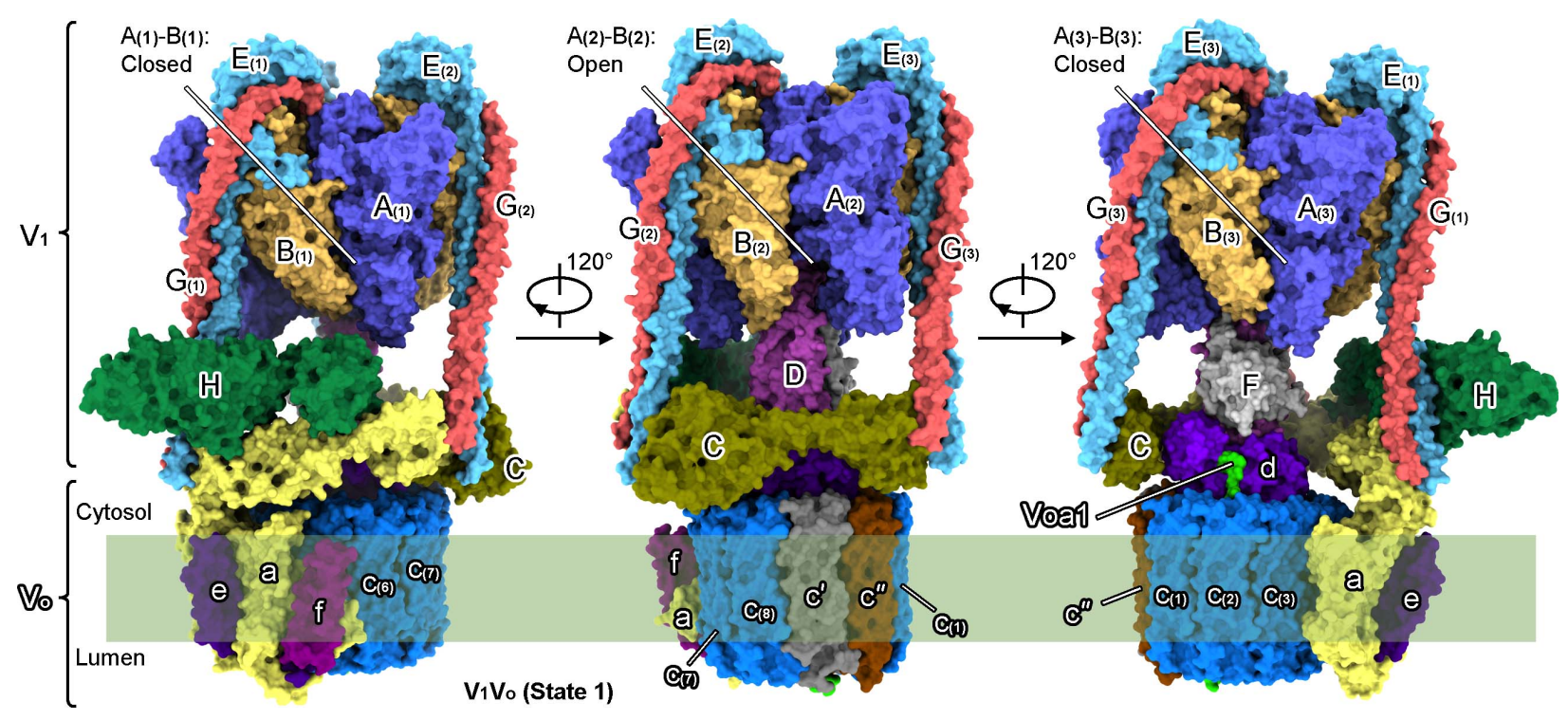

Fig. 1 Structure of the yeast $V_{1} V_{0}$ holoenzyme in state 1. Surface presentation of the yeast $\mathrm{V}_{1} \mathrm{~V}_{\mathrm{o}}$ holoenzyme in state 1 shown with each individual subunit colored and indicated. $\mathrm{V}_{1}$ subunits (in solid uppercase) include three copies of subunit A, B, E, and G, and one copy of subunit $\mathrm{C}, \mathrm{D}, \mathrm{F}$, and $\mathrm{H}$. $\mathrm{V}_{\mathrm{o}}$ subunits (in hollow lowercase) include one copy of subunit a, $\mathrm{c}^{\prime}, \mathrm{c}^{\prime \prime}, \mathrm{d}$, $\mathrm{e}, \mathrm{f}$, and Voa1, and eight copies of subunit c. All subunits are colored using the same color scheme in all figures unless otherwise noted. The catalytic subunit pairs of $A_{(1)}-B_{(1)}$ and $A_{(3)}-B_{(3)}$ are in closed state while $\mathrm{A}_{(2)}-\mathrm{B}_{(2)}$ is open. 


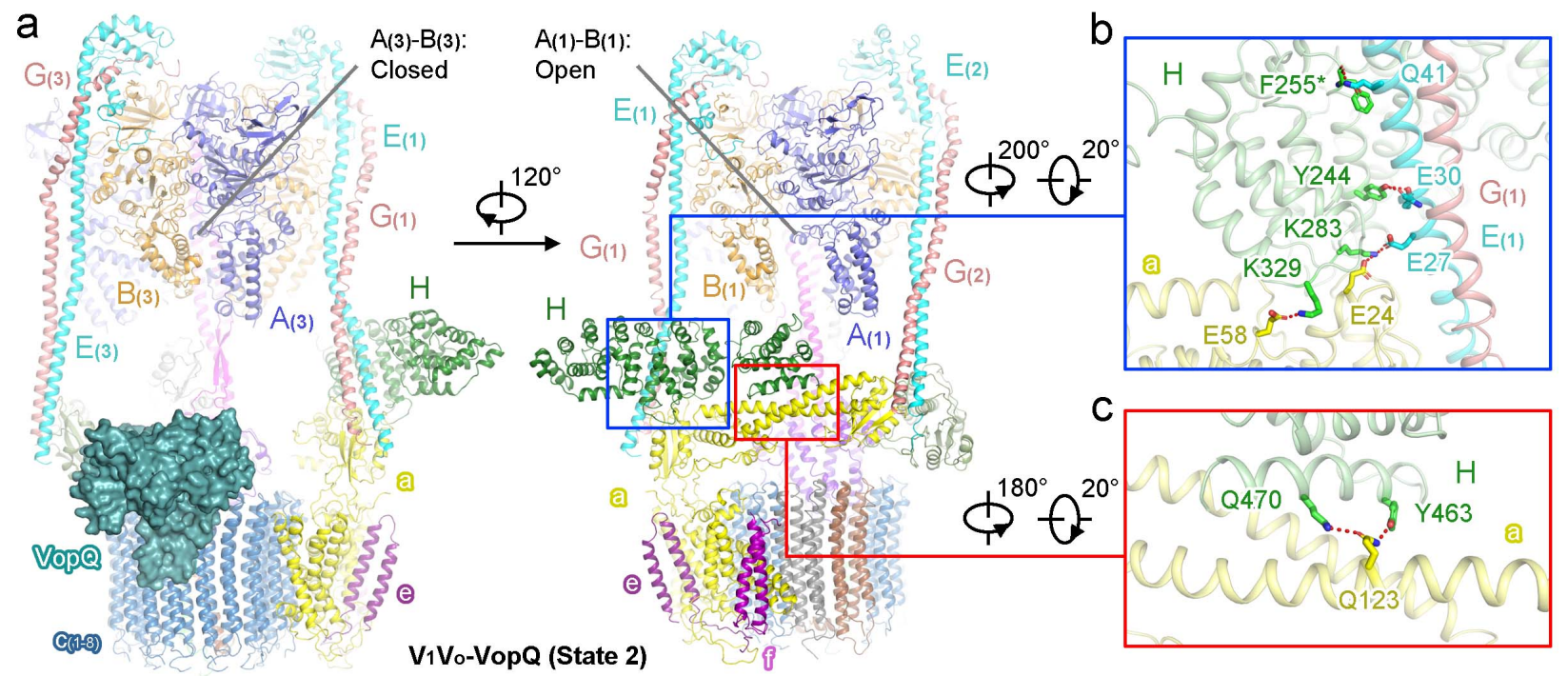

Fig. 2 Structure of the yeast $V_{1} V_{0}-V o p Q$ complex in state 2. a, The yeast $V_{1} V_{0}-V_{0 p Q}$ complex in state 2. VopQ is shown as surface. Subunit $H$ is in close contact with $\mathrm{V}_{1}$ subunit $\mathrm{E}_{(1)}$, $\mathrm{G}_{(1)}$, and $\mathrm{V}_{\mathrm{o}}$ subunit a. b,c, Interaction between subunit $\mathrm{H}$ and subunit $\mathrm{G}$ or subunit a. Residues involved in hydrogen bond or salt bridge interaction are shown as sticks. The main chain carbonyl oxygen atom of F255 in subunit $\mathrm{H}$, indicated by “*”, forms a hydrogen bond with Q41 in subunit $\mathrm{E}_{(1)}$. Both the side chain and main chain oxygen atoms of $\mathrm{E} 30$ in subunit $\mathrm{E}_{(1)}$ are involved in the interaction with Y244 in subunit H. All other interaction pairs are mediated by sides chains as indicated. 


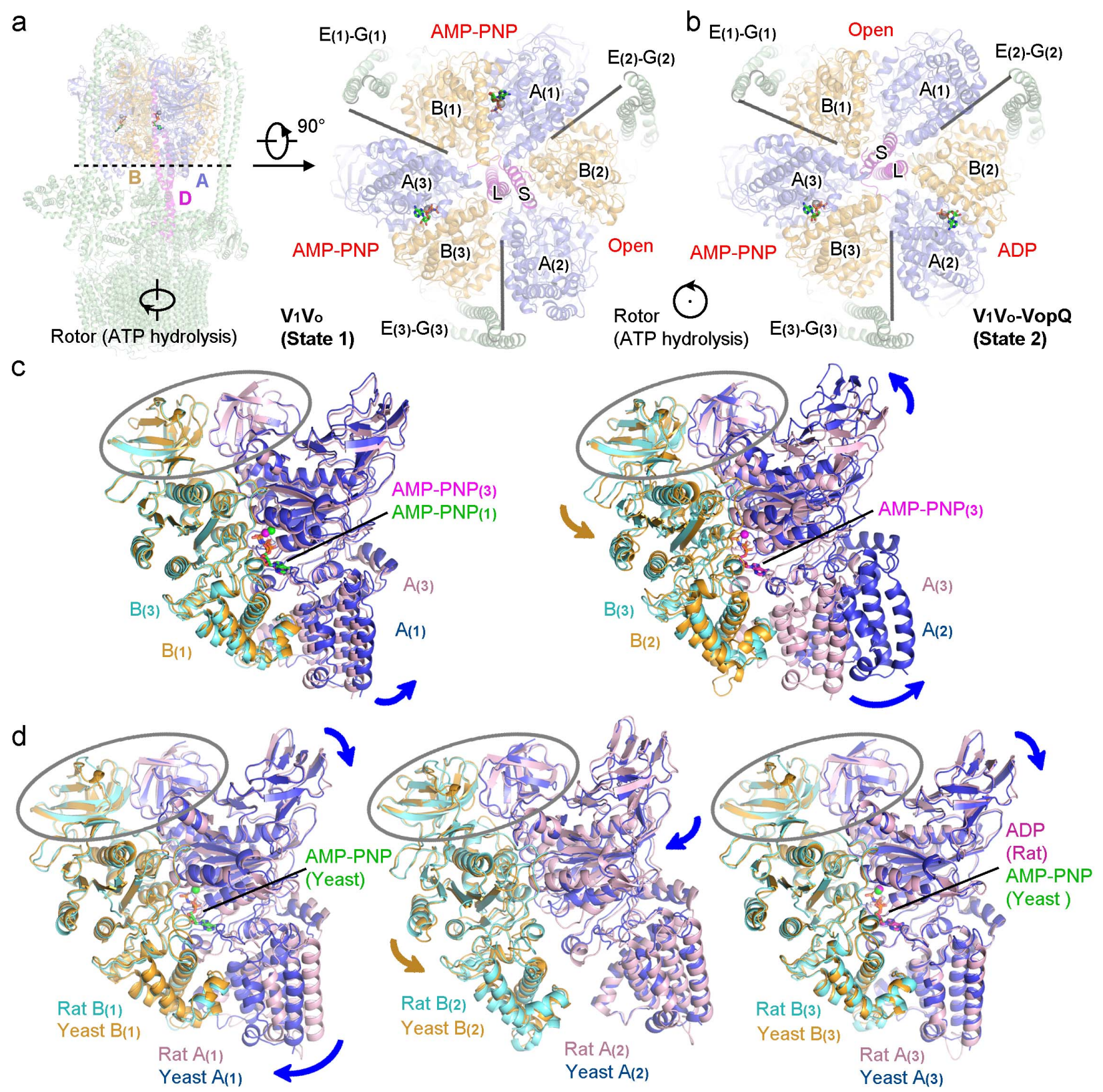

Fig. 3 | Conformations of yeast $V_{1} V_{o}$ catalytic subunit pairs. a,b, The yeast $V_{1} V_{o}$ complex in state 1 and the yeast $\mathrm{V}_{1} \mathrm{~V}_{0}-\mathrm{V}_{0} \mathrm{Qp}$ complex in state 2 . The three catalytic subunit pairs are indicated and separated by radial lines. Subunit D and catalytic subunits are colored and indicated with all the other subunits in green. Nucleotide molecules are shown as sticks with magnesium ions as spheres. "S" or "L", short N-terminal helix or long C-terminal helix of subunit D. c, Comparison of subunit $A-B$ pairs of the yeast $V_{1} V_{o}$ complex in state 1 . d, Comparison of subunit A-B pairs in the yeast $\mathrm{V}_{1} \mathrm{~V}_{\mathrm{o}}$ complex (state 1) and in the rat complex (state 1). Catalytic pairs are aligned against the $\mathrm{N}$-terminal $\beta$-barrel domains indicated by ovals. Relative motions are indicated by colored arrows. 


\section{Supplementary Figures and Legends}

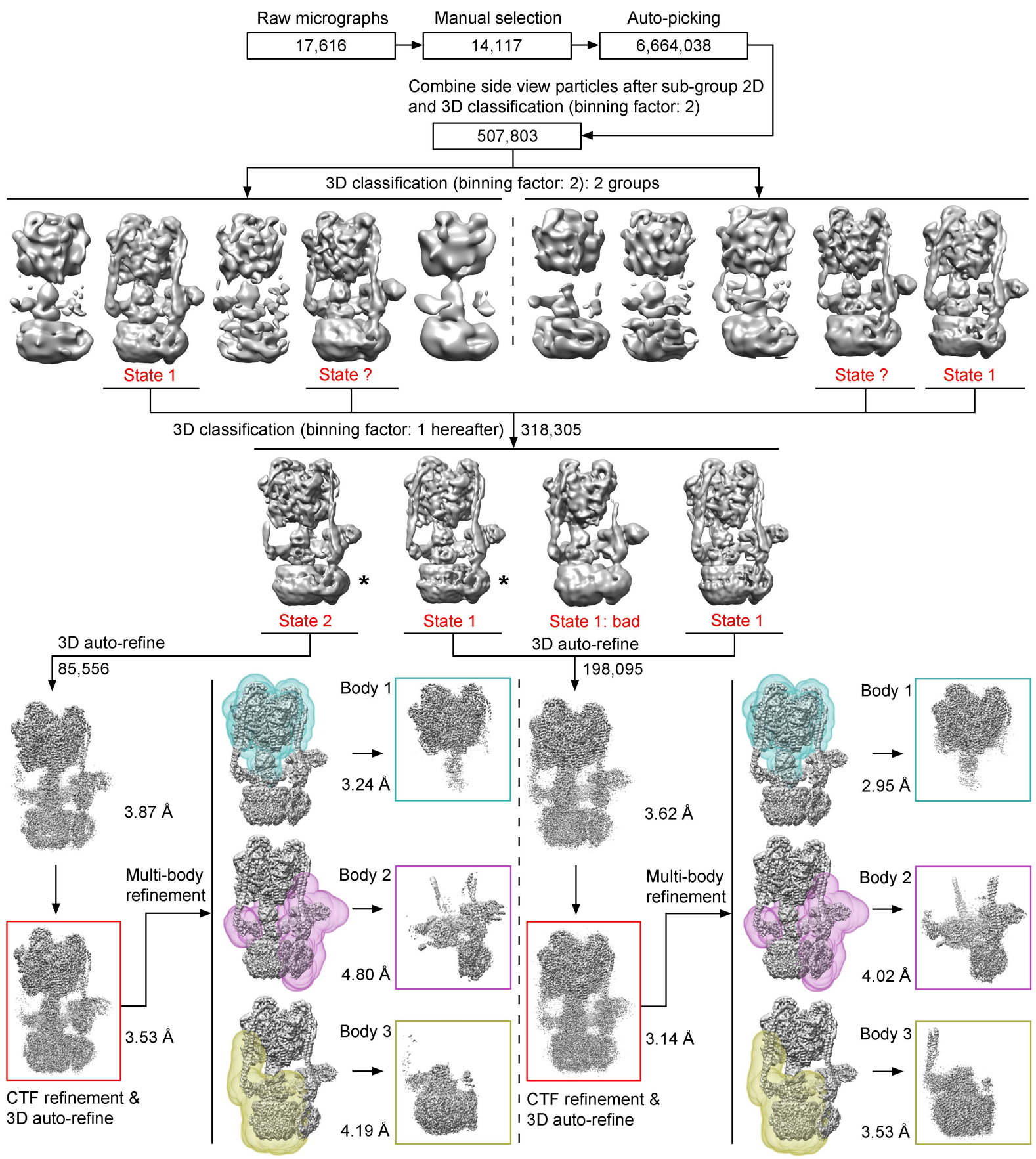

Extended Data Fig. 1 | Flowchart of cryo-EM data processing for the yeast $\mathrm{V}_{1} \mathrm{~V}_{\mathrm{o}}$ holoenzyme. More details are described in methods and shown in Extended Data Fig. 3. 3D classes indicated with “*” are shown in Extended Data Fig. 5a in comparison with $V_{1} V_{0}-V o p Q$ cryo-EM density map. 


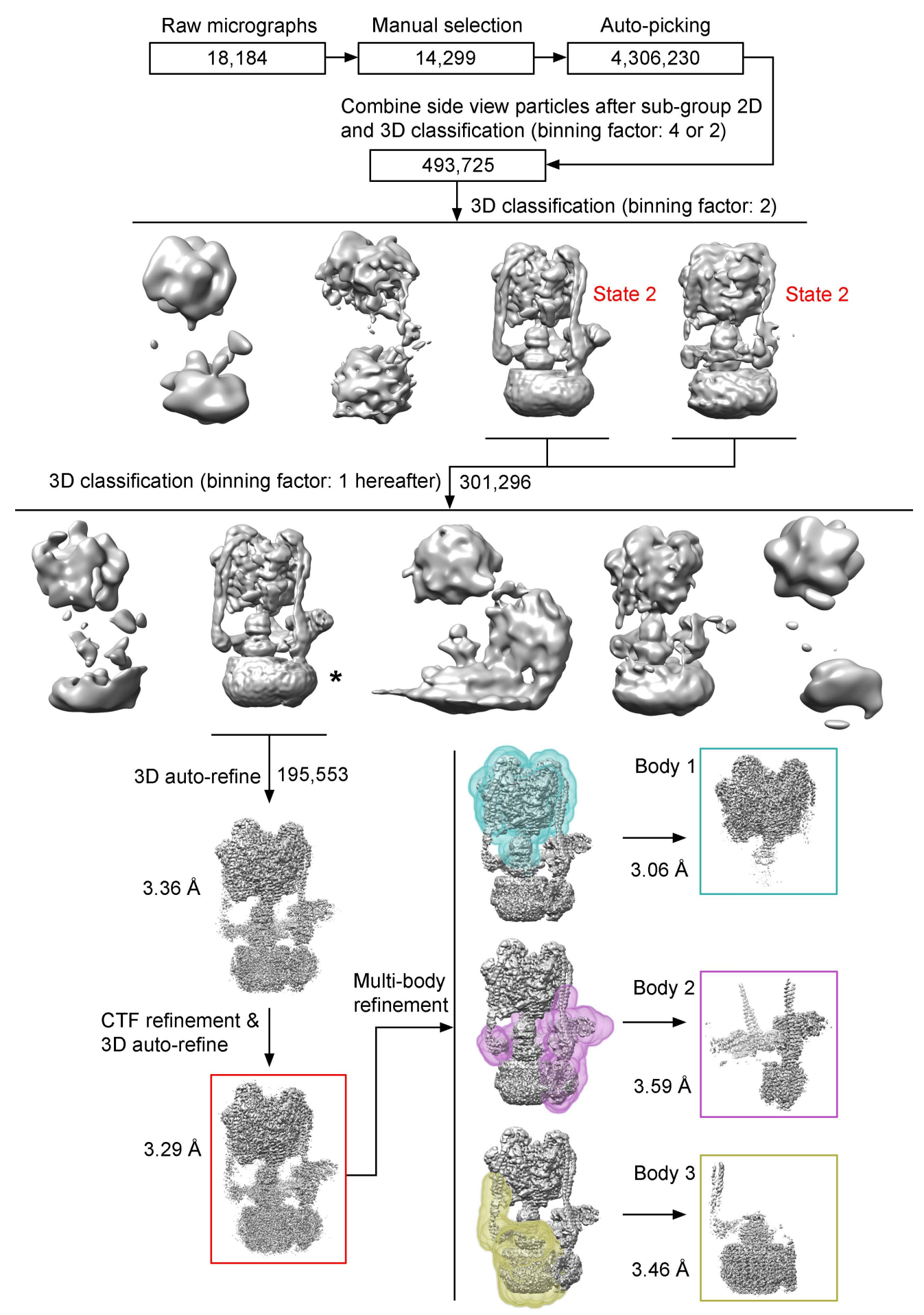
Extended Data Fig. 3. The 3D class indicated with “*” is shown in Extended Data Fig. 5a in 


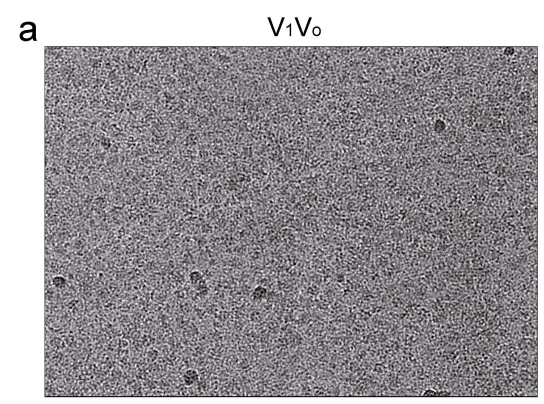

$\mathrm{V}_{1} \mathrm{~V}_{\mathrm{o}}-\mathrm{Vop}_{\mathrm{p}}$

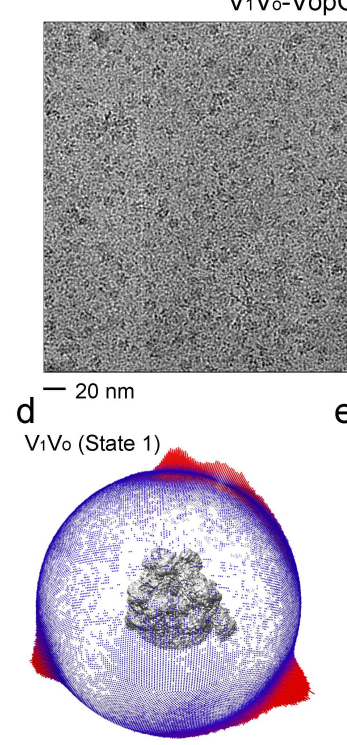

$\mathrm{V}_{1} \mathrm{~V}_{0}($ State 2$)$

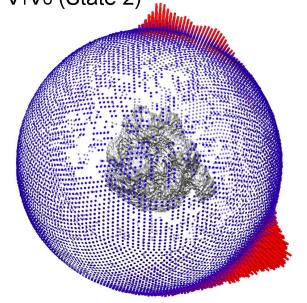

$\mathrm{V}_{1} \mathrm{Vo}_{\mathrm{O}} \mathrm{VopQ}$ (State 2)

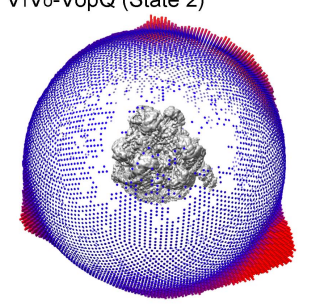

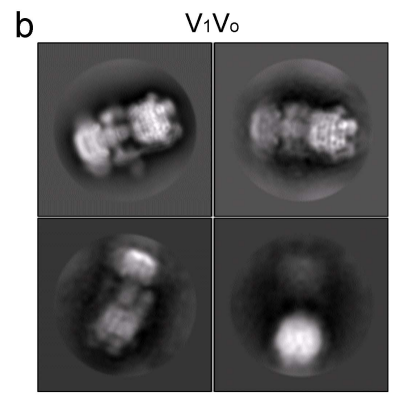

$\mathrm{V}_{1} \mathrm{~V}_{\mathrm{o}}-\mathrm{VopQ}_{\mathrm{O}}$

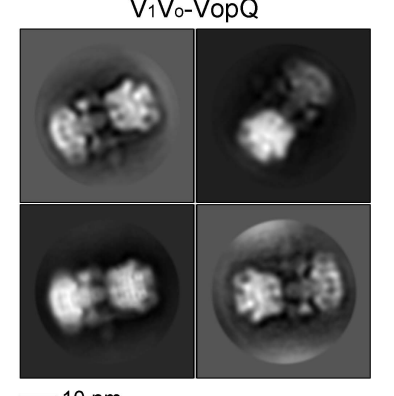

$10 \mathrm{~nm}$
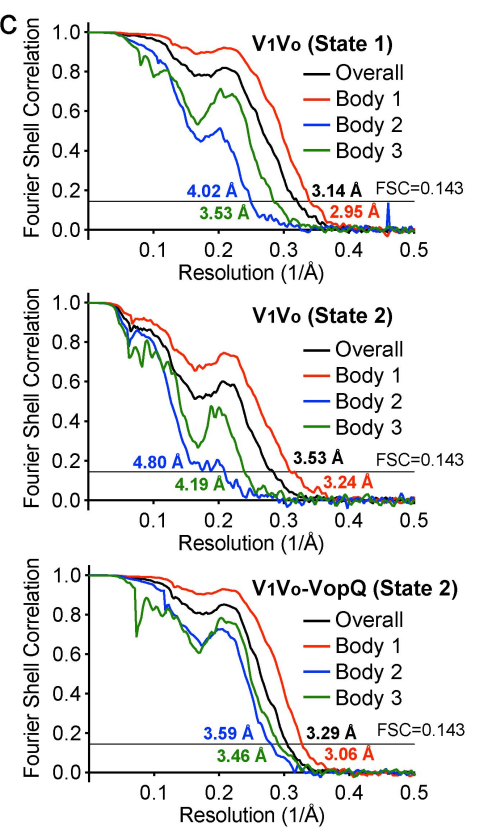

$V_{1} V_{0}$ (State 1)

Body 2

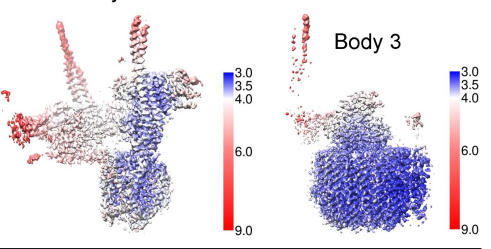

$\mathrm{V}_{1} \mathrm{~V}_{\mathrm{o}}$ (State 2)
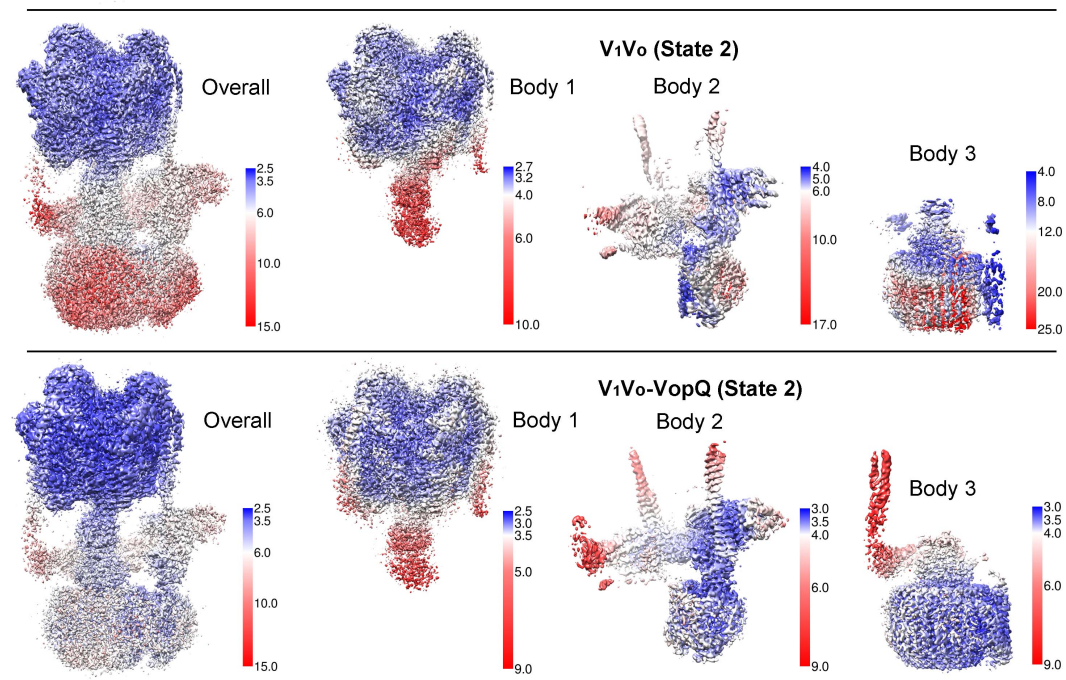

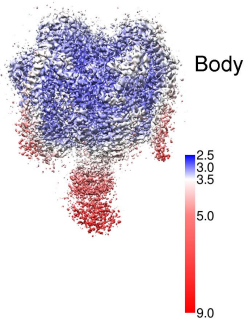

ViVo-VopQ (State 2)

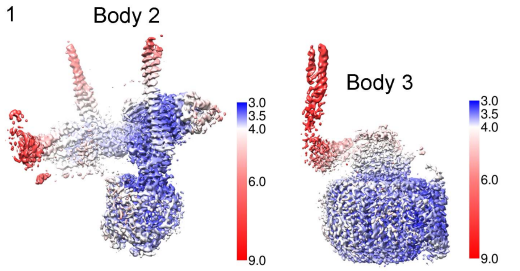

Extended Data Fig. 3 | Analysis of the cryo-EM data. a, Representative cryo-EM micrographs. b, Representative 2D class averages. c, Gold-standard FSC curves for the cryoEM density maps of the yeast $\mathrm{V}_{1} \mathrm{~V}_{\mathrm{o}}$ holoenzyme without and with VopQ bound. Multi-body refinement FSC curves are also presented. d, Angular distributions of particles used in the final $3 \mathrm{D}$ reconstructions, with the heights of the cylinders corresponding to the numbers of particles. 

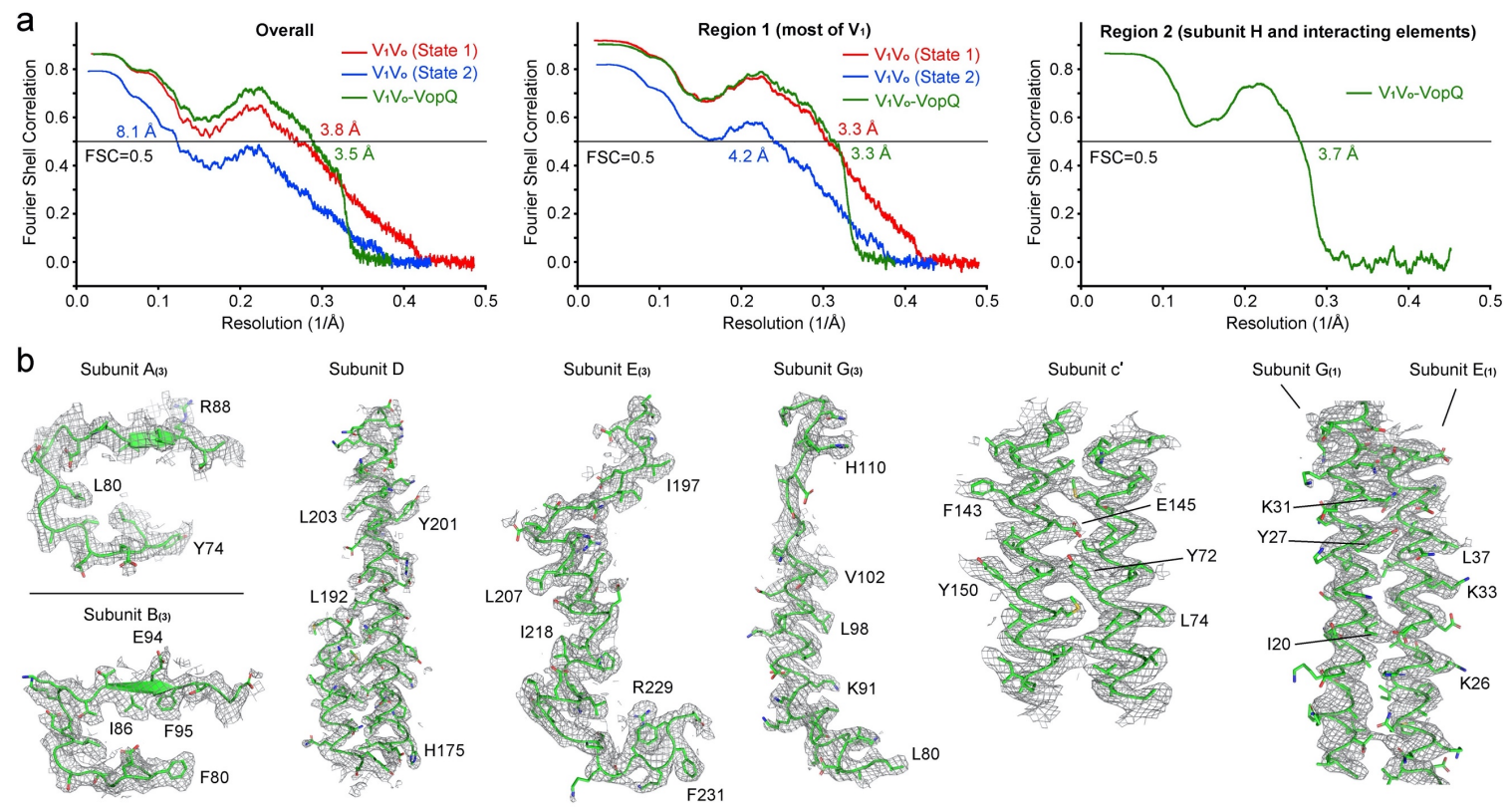

Subunit $\mathrm{H}$
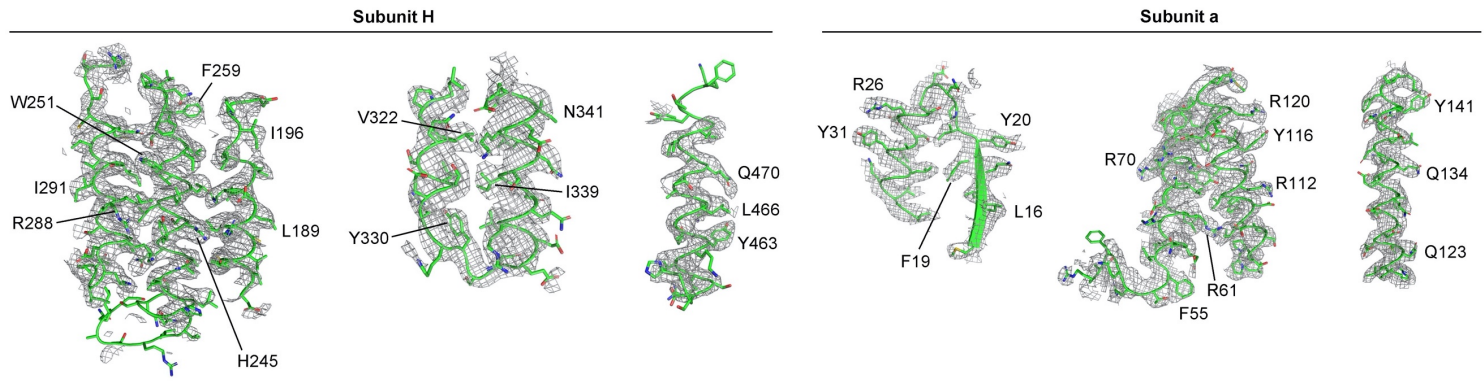

C
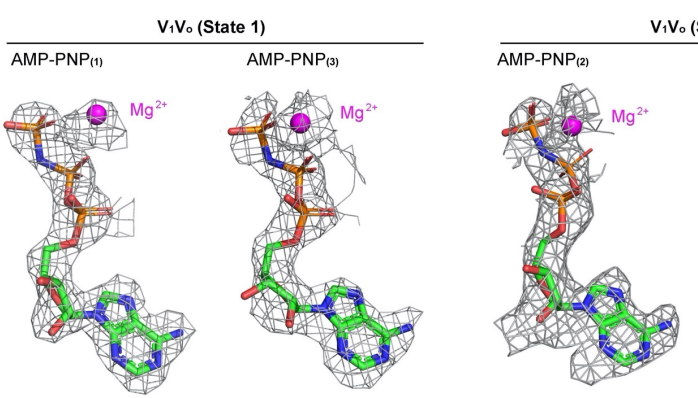

$V_{1} V_{0}$ (State 2)
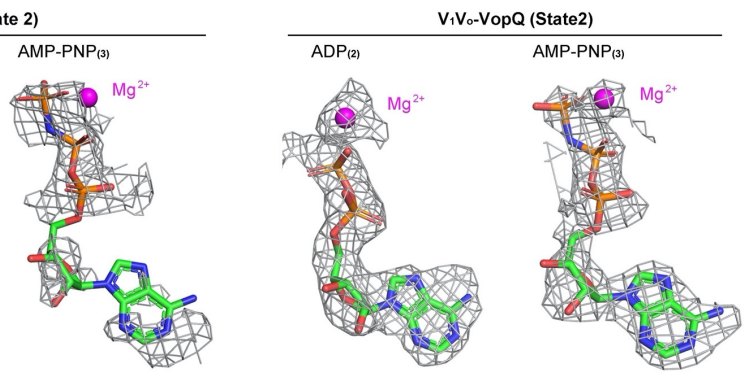

Extended Data Fig. 4 | Model building of the yeast $V_{1} V_{0}$ holoenzyme without or with VopQ bound. a, FSC curves for cross-validation between the final refined models and the maps. Curves for the intact structure models (Overall) against the merged maps, for most of $\mathrm{V}_{1}$ subunits (Region 1 contains subunit $A$ and $B, 61-233$ of subunit E, 61-114 of subunit G, 8-50_91115_151-217 of subunit $\mathrm{D}$, and ligands) against body 1 maps, for subunit $\mathrm{H}$ and neighboring subunits (Region 2 contains subunit H, 8-50 of subunit E, 2-40 of subunit G, and 3-380 of subunit a) against body 2 maps. The $\mathrm{V}_{1} \mathrm{~V}_{\mathrm{o}}$-VopQ model was validated (Overall) without VopQ as the density for VopQ was not well resolved. b, Local density maps shown as mesh for representative elements in the complex of $\mathrm{V}_{1} \mathrm{~V}_{\mathrm{o}^{-}}$-VopQ (contoured at $5.0 \mathrm{\sigma}$ ). Representative bulky residues are indicated. c, Local density maps for ligands of AMP-PNP and ADP (contoured at $10.0 \sigma)$. 

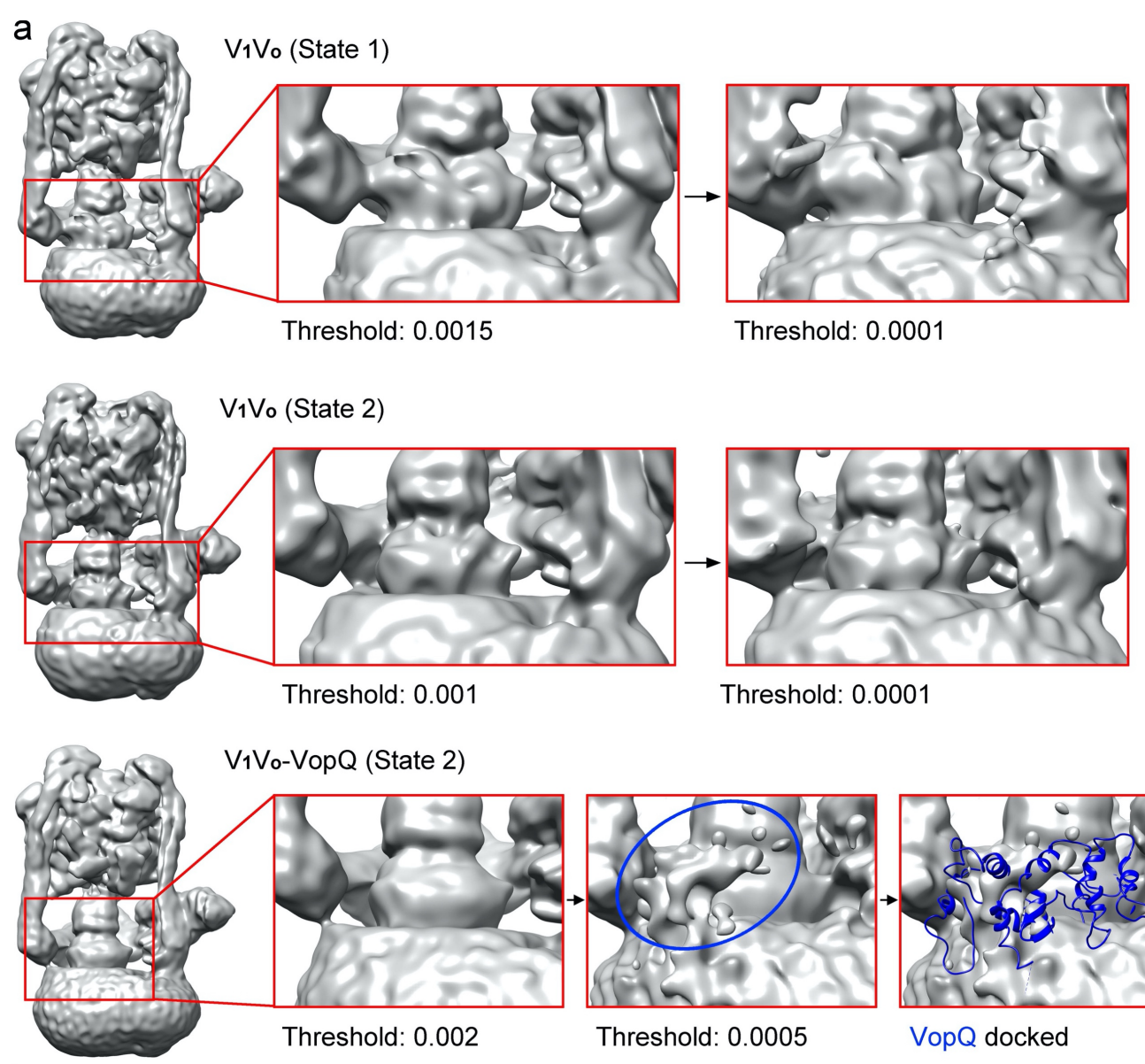

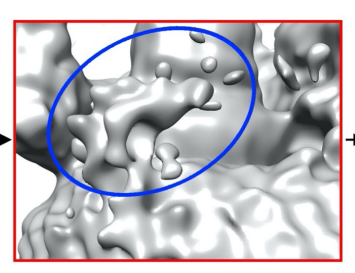

Threshold: 0.0005

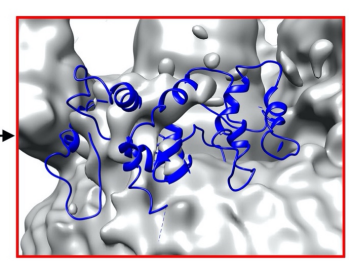

VopQ docked

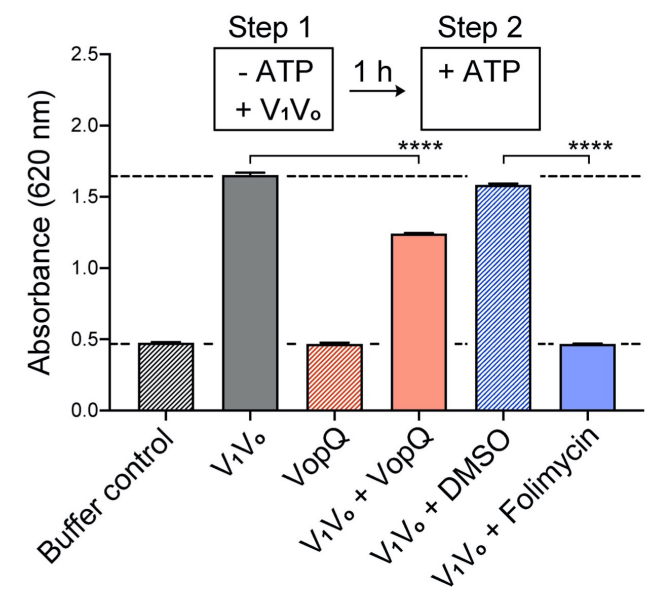

Extended Data Fig. 5 | VopQ can bind the yeast $V_{\mathbf{1}} V_{\mathbf{0}}$ holoenzyme. a, Comparison of the 3D class cryo-EM density maps of the yeast $\mathrm{V}_{1} \mathrm{~V}_{\mathrm{o}}$ holoenzyme without and with VopQ bound. 3D classes indicated in Extended Data Fig. 1-2 are shown at various thresholds. b, VopQ partially inhibits $\mathrm{V}_{1} \mathrm{~V}_{\mathrm{o}}$ ATPase activity in vitro. $\mathrm{V}_{1} \mathrm{~V}_{\mathrm{o}}$ was added into reaction systems with ATP present to initiate ATP hydrolysis (left), or without ATP to bind VopQ before ATP was added (right). Free phosphate produced from ATP hydrolysis was detected by malachite green assay. The VATPase inhibitor, folimycin, was used as a positive control. The baselines of buffer control and full activity are indicated. Data are displayed as mean with SD. Statistical significance was evaluated by using an unpaired Student $\mathrm{t}$ test $(* \mathrm{P}=0.0106 ; * * * * \mathrm{P}<0.0001)$. 
a
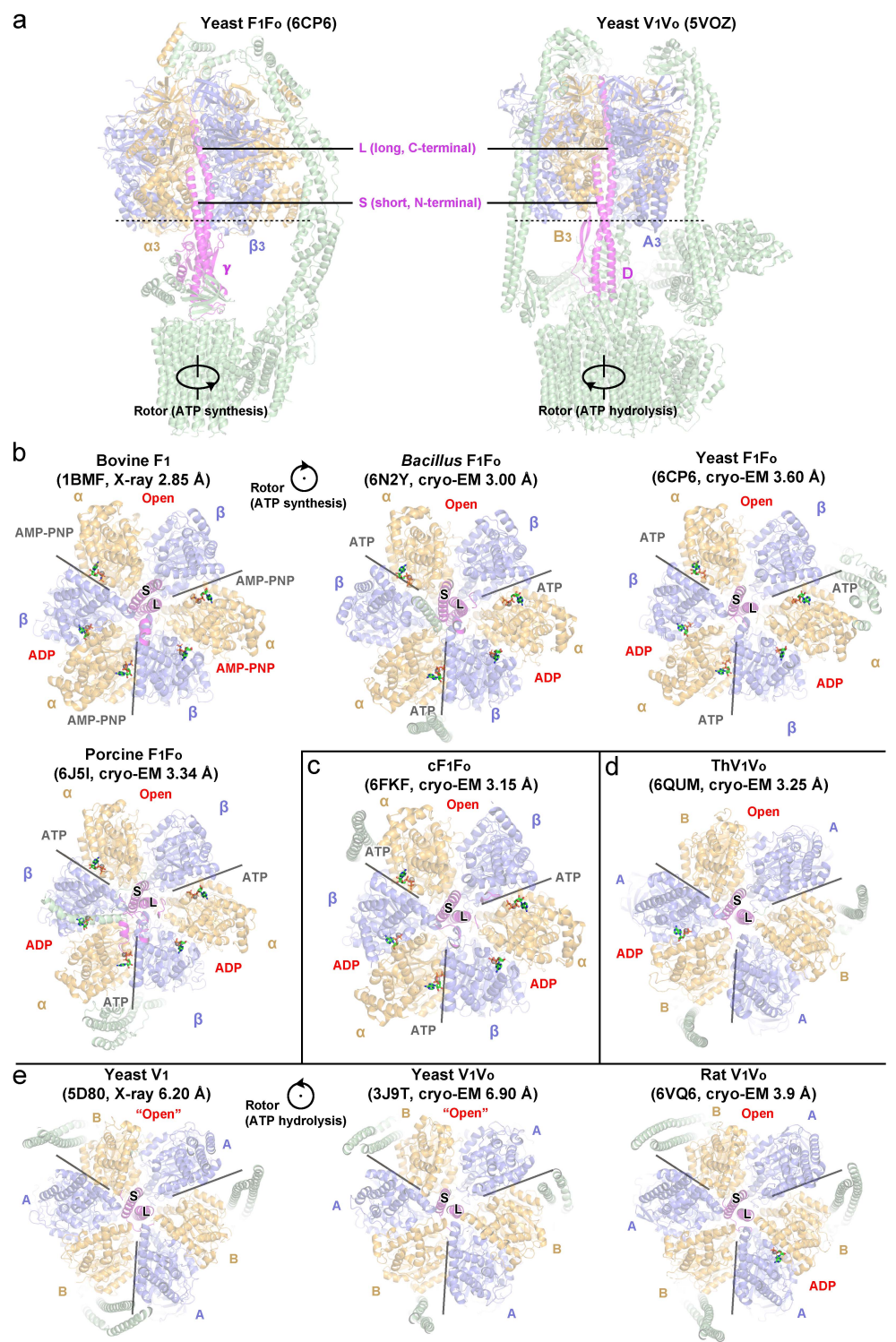

Extended Data Fig. 6 | Representative conformations of rotary ATPases. a, Overall structures of a F-ATPase complex and a V-ATPase complex. Dash lines indicate cross section of catalytic subunits that are shown as bottom views in b-e. b-e, Bottom views of $F_{1}$ and $V_{1}$ catalytic subunits in representative conformations of F-ATPases (b), chloroplast F-ATPase (c), bacterial V/A-ATPase (d), and eukaryotic V-ATPases (e). The three catalytic subunit pairs ( $\beta-\alpha$ in F-ATPases and A-B in V-ATPases) are indicated and separated by radial lines. Subunit $\gamma$ or D and catalytic subunits are colored and indicated with all the other subunits in green. "S" or "L", short N-terminal helix or long C-terminal helix of subunit $\gamma$ and subunit D. Nucleotide molecules are shown as sticks with magnesium ions as spheres. Nucleotides at catalytic sites are labelled red, distinguished from those at non-catalytic sites (only in F-ATPases) in black. PDB accession code for each structure is indicated with the reported resolution noted. F-ATPase contains nonhydrolytic ATP molecules in $\mathrm{F}_{1}$ subcomplex between non-catalytic sites of $\alpha-\beta$ interfaces, but VATPase does not contain non-hydrolytic ATP molecules because of a major defect of P-loop in subunit B. 

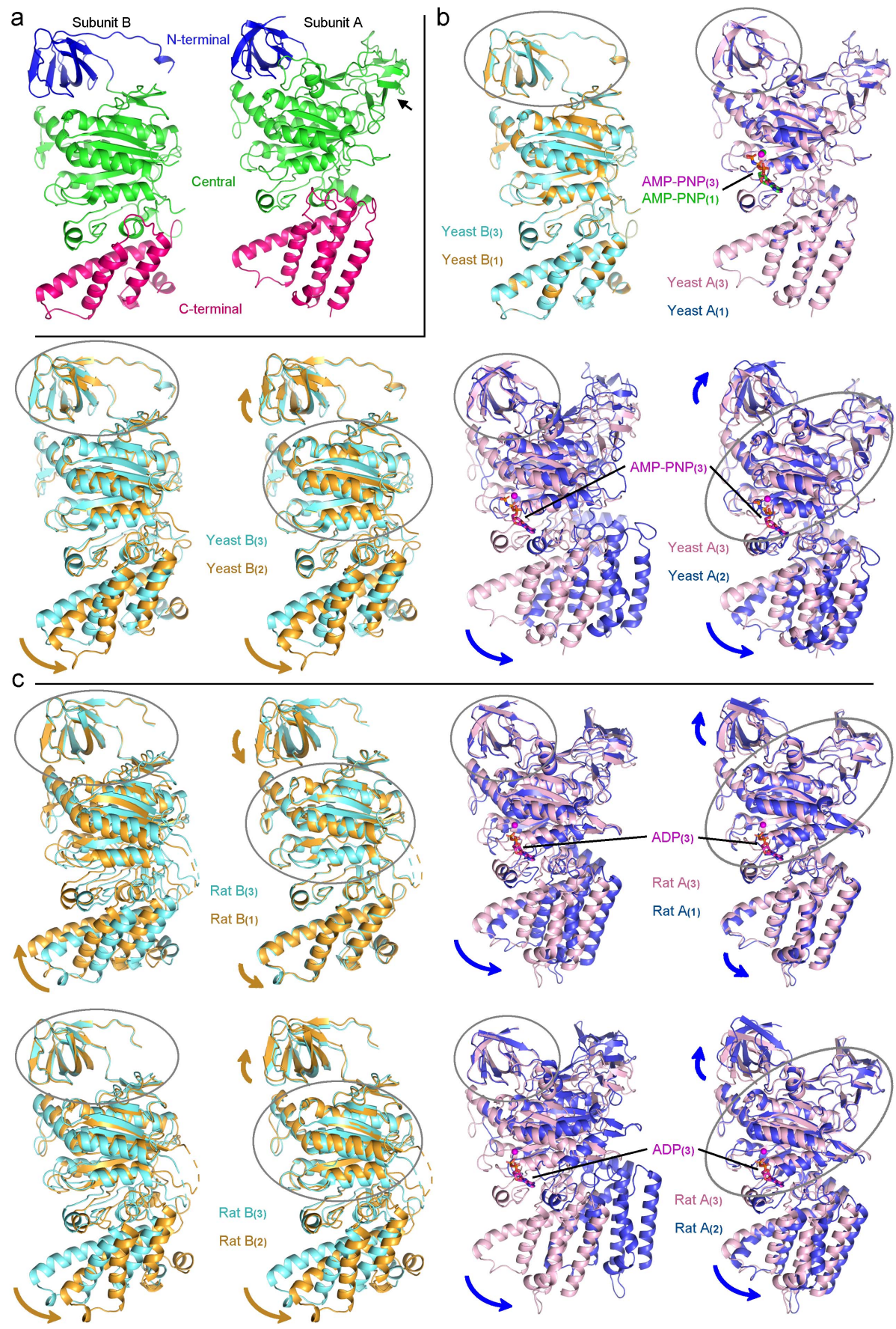

Extended Data Fig. 7 | Comparison of the catalytic subunits in the yeast $V_{1} V_{0}$ complex (state 1) or the rat $V_{1} V_{0}$ complex (state1). a, Domain organization of the yeast catalytic subunits. Each domain is colored as indicated: N-terminal $\beta$-barrel domain (16-98 of B or 24-90 of A), central nucleotide-binding domain (99-390 of B or 91-475 of A), C-terminal domain (391485 of B or 476-617 of A). Subunit A contains a small non-homologous domain (136-213) indicated by an arrow, which is for simplicity classified as part of the central nucleotide-binding domain. b,c, Comparison of subunit $B$ and subunit $A$ in the yeast and the rat $V_{1} V_{o}$ complexes. Subunit $\mathrm{B}_{(3)}$ or $\mathrm{A}_{(3)}$ is aligned against $\mathrm{B}_{(1)}$ or $\mathrm{A}_{(1)}$ and $\mathrm{B}_{(2)}$ or $\mathrm{A}_{(2)}$. The comparison is based on the $\mathrm{N}$-terminal $\beta$-barrel domain or the central nucleotide-binding domain as indicated. 
B-A
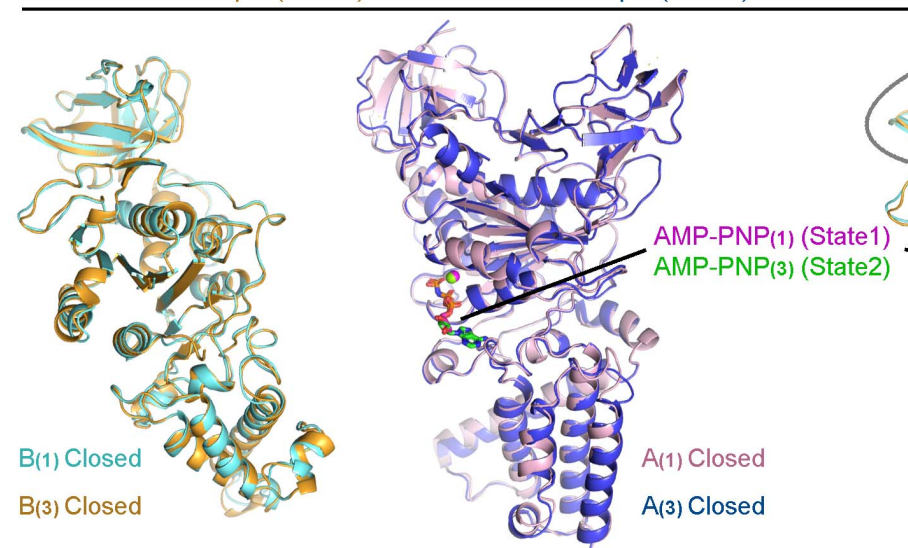
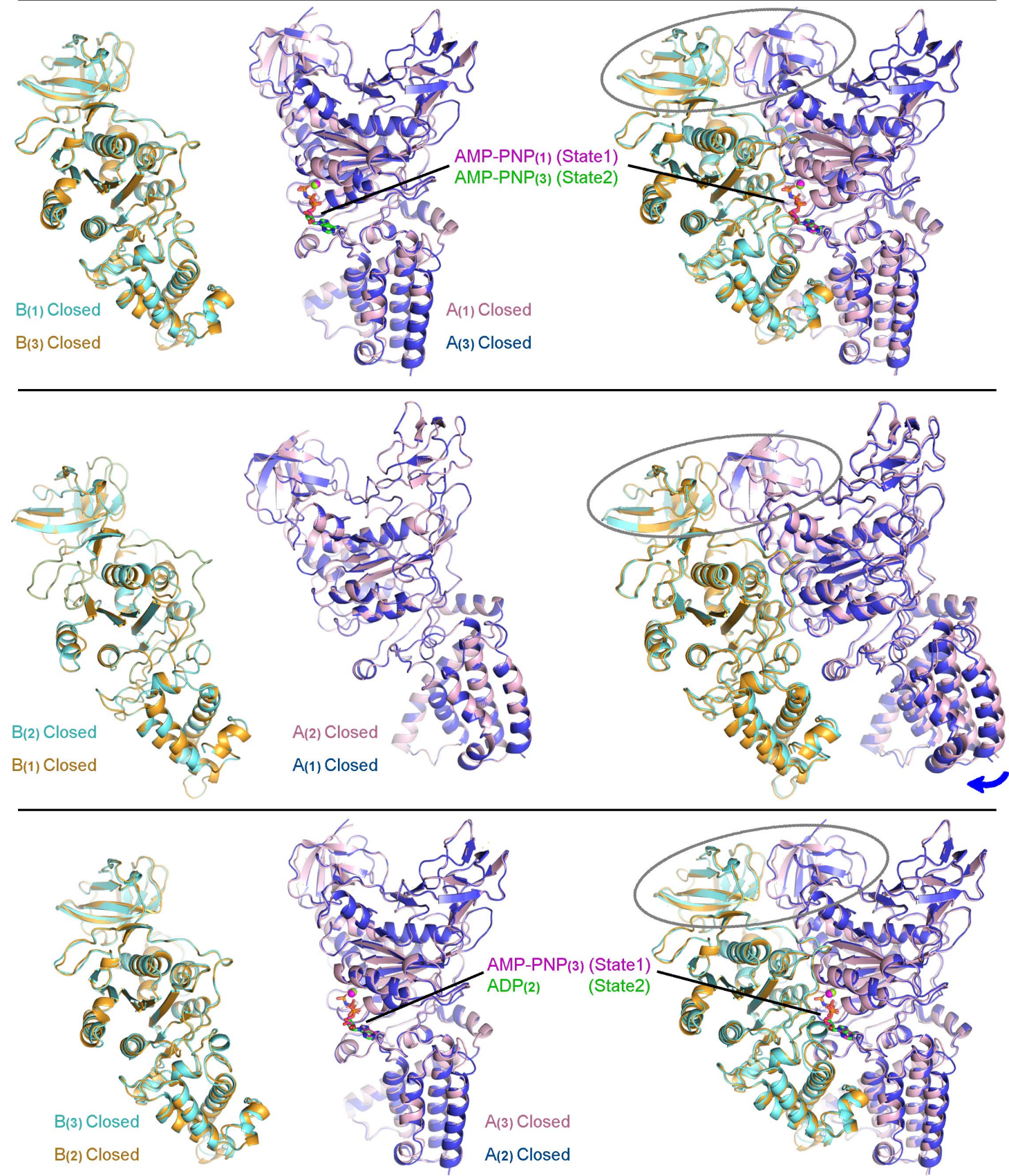

Extended Data Fig. 8 | Comparison of the catalytic subunits in the yeast $V_{1} V_{0}$ complex (state 1) and the yeast $V_{1} V_{0}-V o p Q$ complex (state 2). Individual subunit $B$ or subunit $A$ is aligned against each other from the two complexes. A-B pairs are aligned against the $\mathrm{N}$-terminal $\beta$-barrel domains as indicated. 
Extended Data Table 1. Cryo-EM data collection, refinement and validation statistics.

\begin{tabular}{|c|c|c|c|}
\hline & $\begin{array}{l}\mathrm{V}_{1} \mathrm{~V}_{\mathrm{o}}(\text { State } 1) \\
\text { (EMDB-22298) } \\
\text { (PDB 6XS0) }\end{array}$ & $\begin{array}{l}\mathrm{V}_{1} \mathrm{~V}_{\mathrm{o}}(\text { State } 2) \\
\text { (EMDB-22299) } \\
\text { (PDB 6XS1) }\end{array}$ & $\begin{array}{l}\left.\mathrm{V}_{1} \mathrm{~V}_{\mathrm{o}}-\mathrm{VopQ} \text { (State } 2\right) \\
\text { (EMDB-22300) } \\
\text { (PDB 6XS2) }\end{array}$ \\
\hline \multicolumn{4}{|l|}{ Data collection and processing } \\
\hline Magnification & 59,983 & 59,983 & 59,983 \\
\hline Voltage $(\mathrm{kV})$ & 300 & 300 & 300 \\
\hline Electron exposure $\left(\mathrm{e}^{-} / \AA^{2}\right)$ & $\sim 60$ & $\sim 60$ & $\sim 60$ \\
\hline Defocus range $(\mu \mathrm{m})$ & -1.5 to -2.5 & -1.5 to -2.5 & -1.5 to -2.5 \\
\hline Pixel size $(\AA ̊)$ & 0.83 & 0.83 & 0.83 \\
\hline Symmetry imposed & $\mathrm{C} 1$ & $\mathrm{C} 1$ & $\mathrm{C} 1$ \\
\hline Initial particle images (no.) & $6,664,038$ & $6,664,038$ & $4,306,230$ \\
\hline Final particle images (no.) & 198,095 & 85556 & 195,553 \\
\hline Map resolution $(\AA)$ & $\begin{array}{l}3.14 \\
(2.95 / 4.02 / 3.53)\end{array}$ & $\begin{array}{l}3.53 \\
(3.24 / 4.80 / 4.19)\end{array}$ & $\begin{array}{l}3.29 \\
(3.06 / 3.59 / 3.46)\end{array}$ \\
\hline FSC threshold & 0.143 & 0.143 & 0.143 \\
\hline \multicolumn{4}{|l|}{ Refinement } \\
\hline Initial model used (PDB code) & $6 \mathrm{XS} 2$ & $6 \mathrm{XS} 2$ & 3J9U, 6PE5 \\
\hline Model resolution $(\AA)$ & $2.7 / 3.8$ & $3.1 / 8.1$ & $3.0 / 3.5$ \\
\hline FSC threshold & $0.143 / 0.5$ & $0.143 / 0.5$ & $0.143 / 0.5$ \\
\hline Map sharpening $B$ factor $\left(\AA^{2}\right)$ & $-87.1 /-135.2 /-116.7$ & $-74.2 /-161.5 /-144.2$ & $-102.6 /-132.7 /-127.2$ \\
\hline \multicolumn{4}{|l|}{ Model composition } \\
\hline Non-hydrogen atoms & 64,367 & 64,367 & $64,363(67,075)$ \\
\hline Protein residues & 8278 & 8278 & $8,278(8,647)$ \\
\hline Ligand & 4 & 4 & 4 \\
\hline \multicolumn{4}{|l|}{$B$ factors $\left(\AA^{2}\right)$} \\
\hline Protein & 6.37 & 13.93 & 13.93 \\
\hline Ligand & 1.60 & 12.27 & 3.92 \\
\hline \multicolumn{4}{|l|}{ R.m.s. deviations } \\
\hline Bond lengths $(\AA)$ & 0.012 & 0.010 & 0.010 \\
\hline Bond angles $\left(^{\circ}\right)$ & 1.433 & 1.329 & 1.327 \\
\hline \multicolumn{4}{|l|}{ Validation } \\
\hline MolProbity score & 3.71 & 3.58 & 3.58 \\
\hline Clashscore & 47.19 & 41.97 & 41.63 \\
\hline Poor rotamers $(\%)$ & 17.95 & 16.28 & 16.26 \\
\hline \multicolumn{4}{|l|}{ Ramachandran plot } \\
\hline Favored (\%) & 87.81 & 89.59 & 89.59 \\
\hline Allowed (\%) & 10.69 & 8.97 & 9.01 \\
\hline Disallowed (\%) & 1.50 & 1.44 & 1.40 \\
\hline
\end{tabular}

